\title{
Organic Dust Exposure Induces Stress Response and Mitochondrial Dysfunction in
}

\section{Monocytic Cells}

\section{Authors}

4 Sanjana Mahadev-Bhat ${ }^{1}$, Denusha Shrestha ${ }^{1}$, Nyzil Massey ${ }^{1}$, Locke A. Karriker ${ }^{2}$, Anumantha

5 G. Kanthasamy ${ }^{1}$, Chandrashekhar Charavaryamath ${ }^{1 *}$

\section{Author details}

$7{ }^{1}$ Department of Biomedical Sciences, 2008 Vet Med Building, lowa State University, Ames, IA, 8 USA.

$9{ }^{2}$ Department of Veterinary Diagnostic and Production Animal Medicine, 2203 Lloyd Veterinary 10 Medical Center, Iowa State university, Ames, IA, USA

11 *To whom correspondence should be addressed: Chandrashekhar Charavaryamath, BVSc, 12 MVSc, PhD., Assistant Professor, Department of Biomedical Sciences, lowa State University, 13 Ames, IA 50011. Telephone: (515) 294-7710; Fax: (515) 294-2315; Email: chandru@iastate.edu 14 15 


\section{Abstract}

Exposure to airborne organic dust (OD), rich in microbial pathogen-associated molecular patterns, has been shown to induce inflammatory responses in the lung resulting in changes in airway structure and function. A common manifestation in lung inflammation is the occurrence of altered mitochondrial structure and bioenergetics, consequently regulating mitochondrial ROS (mROS) and creating a vicious cycle of mitochondrial dysfunction.

The role of mitochondrial dysfunction in airway diseases such as COPD and asthma is well known. However, whether OD exposure induces mitochondrial dysfunction largely remains unknown. Therefore, in this study, we tested a hypothesis that OD exposure induces mitochondrial stress using a human monocytic cell line (THP-1). We examined the mechanisms of organic dust extract (ODE) exposure-induced mitochondrial structural and functional changes in THP-1 cells.

In addition, the effect of co-exposure to ethyl pyruvate (EP), a known anti-inflammatory agent, or mitoapocynin (MA), a mitochondria targeting NOX2 inhibitor was examined. Transmission electron microscopy images showed significant changes in cellular and organelle morphology upon ODE exposure. ODE exposure with and without EP co-treatment increased the mtDNA leakage into the cytosol. Next, ODE exposure increased the PINK1 and Parkin expression, cytoplasmic cytochrome c levels and reduced mitochondrial mass and cell viability, indicating mitophagy. MA treatment was partially protective by decreasing Parkin expression, mtDNA and cytochrome c release and increasing cell viability.

Keywords: Organic dust, mitoapocynin, ethyl pyruvate, mitochondrial dysfunction 


\section{Introduction}

57 Industrialized agriculture production systems form the backbone of the farm economy in the USA 58 with a large number of workforce and a major contribution to the nation's GDP (Charavaryamath and Singh, 2006; Nordgren and Charavaryamath, 2018; Sethi et al., 2017). Despite the production efficiency and cheaper price of the food, these industries have occupational hazards in the form of exposure to many on-site contaminants. Among the contaminants, airborne organic dust (OD) and gases (mainly hydrogen sulfide, methane and ammonia), viable bacteria, fungal spores and other microbial products are known to be present ("Respiratory Health Hazards in Agriculture," 1998). OD comprises of particulate matter (PM) of varying sizes from plant, animal and microbial sources (Vested et al., 2019). Bacterial lipopolysaccharide (LPS) and peptidoglycan (PGN) are the major microbial pattern recognition receptors (PAMPs) present in the OD samples. Agriculture production workers who are exposed to OD report several respiratory symptoms and annual decline in the lung function (Nordgren and Charavaryamath, 2018; Sethi et al., 2017; Wunschel and Poole, 2016). Strategies that reduce the levels of dust in the workplace have shown to have positive health impacts (Senthilselvan et al., 1997).

Respiratory symptoms of exposed workers include bronchitis, coughing, sneezing, chesttightness, asthma and asthma like symptoms, mucus membrane irritation and other signs. Persistent exposure to OD has been linked to the development of chronic inflammatory conditions, such as chronic obstructive pulmonary disease (COPD) and asthma, including lung tissue damage and decline in lung function (Charavaryamath and Singh, 2006; Wunschel and Poole, 2016). Despite several research groups using both in vitro and in vivo models of OD exposure, precise cellular and molecular mechanisms leading to chronic lung disease remain largely unknown.

In response to this, an understanding of the mechanisms of induction of airway inflammation is essential as it promotes the development of strategies for the maintenance of lung homeostasis by preserving the balance between pro-inflammatory and anti-inflammatory responses. Studies have shown that OD-mediated lung inflammation is typically characterized by airway hyperresponsiveness (AHR), tissue remodeling, and increased influx of inflammatory cells, particularly neutrophils and macrophages, in lung tissues (Charavaryamath et al., 2005; Sahlander et al., 2012; Sethi et al., 2017). In previous studies we have shown exposure of human bronchial epithelial cells to OD results in the production of reactive oxygen species (ROS), reactive nitrogen species (RNS) and a myriad of pro-inflammatory cytokines such as interleukins 
also becoming increasingly clear that, in addition to the above factors, abnormal mitochondrial signatures and mitochondrial dysfunction contribute to the pathological mechanisms of lung disease (Cloonan and Choi, 2016). Several in vitro and in vivo studies have demonstrated the elevation of key enzymes involved in the production of ROS and RNS due to mitochondrial impairment in various inflammatory conditions (Cloonan and Choi, 2012; Eisner et al., 2018; Zhang et al., 2010). Collectively, these findings suggest that targeting multiple pathogenic mechanisms, including mitochondrial impairment, oxidative stress, and other inflammatory processes, could provide an advantage over targeting a single disease pathway.

Mitochondria are dynamic double membraned organelles that possess their own genome and proteome. These are ubiquitously present and are critical for many of the body's "housekeeping" functions, including synthesis and catabolism of metabolites, calcium regulation, and most importantly generation of ATP by oxidative phosphorylation (OXPHOS) (Tilokani et al., 2018). Whilst the participation of mitochondria in OXPHOS, stress responses and programmed cell death pathways have been well studied over the past decade, the role of mitochondria in the activation and control of the immune response has been of interest. During inflammation, mitochondria can become damaged or dysfunctional leading to impaired cellular respiration and cell death. The presence of dysfunctional mitochondria can lead to oxidative stress which acts as a potent stimulus for exacerbating inflammation (Cloonan and Choi, 2012; Eisner et al., 2018).

The adverse effects of inflammation on mitochondria can be abrogated by several mechanisms. These include the induction of anti-inflammatory responses and antioxidant defenses, maintenance of mitochondrial integrity through the selective removal of dysfunctional mitochondria (mitophagy), and the generation of new organelles to replace them (mitochondrial biogenesis) (Eisner et al., 2018). However, the integration of these compensatory responses, and the interaction between mitochondria and host cells following OD exposure, are not well understood. In order to study these processes, we assessed mitochondrial functions, biogenesis, and mitophagy on exposure to OD alone and in the presence of antioxidant therapies, such as ethyl pyruvate (EP) and mitoapocynin (MA), which have previously been shown to have significant antioxidative functions.

The protective effects of EP have been attributed to its anti-inflammatory, antioxidative and antiapoptotic action. Previously we have shown the effectiveness of ethyl pyruvate (EP) as a nonspecific inhibitor of inflammatory cytokine-like high mobility group box 1 (HMGB1) release into the extracellular space in bronchial epithelial cells (Bhat et al., 2019). We also demonstrated that EP downregulates reactive oxygen species (ROS) generation and augmented IL-10 production thus 
122 promoting anti-inflammatory effects. Similar results have been shown in LPS injected and

123 ischemic animal models (Venkataraman et al., 2002; Yu et al., 2005). The anti-inflammatory

124 property of EP has been attributed to the inhibition of ROS-dependent signal transducer and

125 activator of transcription (STAT) signaling (Kim et al., 2008).

126 In addition to using EP, we also tested the efficacy of MA in a OD-induced inflammatory model.

127 In previous studies, apocynin, a plant derived antioxidant, has been used as an efficient inhibitor

128 of NADPH-oxidase complex in many experimental models involving phagocytic and

129 nonphagocytic cells (Stefanska and Pawliczak, 2008). In this study we used

130 triphenylphosphonium (TPP) conjugated apocynin (mitoapocynin, MA) designed to enhance their

131 cellular uptake and target the mitochondria. In contrast to other popular antioxidant therapies, MA

132 has been shown to attenuate ROS and/or RNS generation in both in vitro and in vivo models of

133 neuroinflammation. In an MPTP-induced neuroinflammatory model, MA treatment was shown to

134 suppress iNOS and various pro-inflammatory cytokines. In addition, MA was shown to inhibit

135 NOX2 activity and reduce oxidative stress (Ghosh et al., 2016; Langley et al., 2017).

136 In this study we used an immortalized human monocytic cell line (THP1) and tested a hypothesis

137 that OD-exposure induces mitochondrial stress. We further examined whether there is an

138 induction of antioxidant defenses, changes in mitophagy and mitochondrial biogenesis in THP1

139 cells following exposure to OD in the presence of both a mitochondrial specific NOX2 inhibitor

140 (MA) and an inhibitor of HMGB1 translocation (EP), leading to the maintenance of cellular viability

141 and mitochondrial integrity. Here we demonstrate that mitochondrial specific or general

142 antioxidant therapy, through inhibition of HMGB1 translocation, are vital to cellular recovery

143 following exposure to OD.

144 Materials and Methods

145 Chemicals and reagents

146 We purchased RPMI 1640, L- glutamine, penicillin, streptomycin, MitoTracker green, and 147 MitoSOX Red stains from Invitrogen (ThermoFisher Scientific) and fetal bovine serum (FBS) was 148 purchased from Atlanta Biologicals. Antibodies for mitofusins (MFN1/2), DRP1, PINK1, Parkin, 149 OPA1, BNIP3, Cytochrome C, COX4i2, Bcl-2, Bcl-XL, mtTFA, Caspase 1 and Caspase 3 was 150 purchased from Santa Cruz Biotechnology. The anti-HMGB1 antibody, $\beta$-Actin antibody and 151 Rhod-2AM dye were obtained from Abcam. MitoApocynin- $C_{11}$ (MA) was procured from Dr. 152 Balaraman Kalyanaraman (Medical College of Wisconsin, Milwaukee, WI), stock solution (10 mM 153 in DMSO) prepared by shaking vigorously and stored at $-20^{\circ} \mathrm{C}$. MA was used (10 $\left.\mu \mathrm{M}\right)$ as one of 
154

155

156

157

158

159

160

161

162

163

164

165

166

167

168

169

170

171

172

173

174

175

176

177

178

179

180

181

182

183

the co-treatments. Ethyl pyruvate (EP) purchased from Santa Cruz Biotechnology, was reconstituted in Ringer's solution (Sigma-Aldrich) and used at a final concentration of $2.5 \mu \mathrm{M}$ in the cell culture medium.

\section{Organic dust extract preparation}

Aqueous organic dust extract (ODE) was collected and prepared as previously described (Bhat et al., 2019; Romberger et al., 2002). Briefly, settled surface dust samples from swine housing facilities were collected and $1 \mathrm{~g}$ was placed into sterile Hank's Balanced Salt Solution (10 ml; Gibco). Solution was incubated for one hour at room temperature, centrifuged for 20 min at 1365 $x \mathrm{~g}$, and the final supernatant was filter sterilized $(0.22 \mu \mathrm{m})$, a process that also removes coarse particles. Stock (100\%) ODE aliquots frozen at $-20^{\circ} \mathrm{C}$ until use in experiments. The filter sterilized organic dust extract (ODE) samples were considered $100 \%$ and diluted to $1-5 \%(v / v)$ before use in experiments.

\section{Cell culture and treatments}

Immortalized human monocytic cells (THP1, ATCC TIB-202 ${ }^{\mathrm{TM}}$ ) were used in this study. These cell lines have previously been used to study innate inflammatory responses to ODE (Nath Neerukonda et al., 2018). THP1 cells were cultured in RPMI 1640 at $37^{\circ} \mathrm{C}$ in a humidified chamber with 5\% $\mathrm{CO}_{2}$. The RPMI 1640 medium was supplemented with 10\% (v/v) heat-inactivated FBS, $2 \mathrm{mM}$ L-glutamine, $10 \mathrm{mM}$ HEPES, $1.5 \mathrm{~g} / \mathrm{L}$ sodium bicarbonate, $1 \mathrm{mM}$ sodium pyruvate, $100 \mathrm{IU} / \mathrm{ml}$ penicillin, and $100 \mu \mathrm{g} / \mathrm{ml}$ streptomycin (Gibco) and $1 \mu \mathrm{g} / \mathrm{mL}$ of Amphotericin B (Sigma-Aldrich). Cells were subcultured once a week and the morphology was observed. Approximately 4 to 5day old cultures were used for experiments. Treatments were done in 1\% FBS-containing medium for 24 hours. All groups with treatment details are outlined in figure 1.

Ethyl pyruvate (EP) was reconstituted in Ringer's solution and used at a final concentration of 2.5 $\mu \mathrm{M}$ in the cell culture medium. Mitochondrial specific drug mitoapocynin (MA) was diluted in dimethyl sulfoxide (DMSO) and used at a final concentration of $10 \mu \mathrm{M}$ (Ghosh et al., 2016; Langley et al., 2017).

Cells were treated with either medium (control) or ODE $(1 \% \mathrm{v} / \mathrm{v})$ followed by a co-treatment with either EP $(2.5 \mu \mathrm{M})$ or MA $(10 \mu \mathrm{M})$ for 24 hours, with corresponding time matched controls. Following the treatments, samples were processed at 24 hours for various assays.

\section{Table 1. Stock and working concentrations of treatments}




\begin{tabular}{ccc}
\hline Treatments & Stock concentration & $\begin{array}{c}\text { Working concentration (in } \\
\text { 1\% FBS-containing } \\
\text { medium) }\end{array}$ \\
ODE & $100 \%$ in HBSS & $1 \%$ \\
EP & $5 \mathrm{mM}$ in ringer's solution & $2.5 \mu \mathrm{M}$ \\
MA & $1 \mathrm{mM}$ in DMSO & $10 \mu \mathrm{M}$ \\
\hline
\end{tabular}

Prior to conducting experiments, cell viability was assessed. Live/dead cell count was determined by $4 \%$ trypan blue dye (EMD Millipore) exclusion and percentage viability was calculated. Population of cells with more than $95 \%$ viability were used for the experiments.

The MTT assay has been widely used in the estimation of LC50 and cell viability by measuring the formazan produced when mitochondrial dehydrogenase enzymes cleave the tetrazolium ring 191 (Latchoumycandane et al., 2005). In this study, we used the MTT assay to determine the LC50 192 of ODE in THP1 cells. Cells were seeded (20,000 cells/well) in a 96-well culture plate and treated 193 with ODE for 24 hours in 1\% FBS-containing RPMI medium. After the treatment, the cells were 194 washed with PBS and incubated with $0.5 \mathrm{mg} / \mathrm{mL}$ of MTT in 1\% FBS-containing RPMI medium for 1953 hours at $37^{\circ} \mathrm{C}$. The supernatant was removed, and MTT crystals were solubilized in $100 \mu \mathrm{l}$ of 196 DMSO. Absorbance was measured with the SpectraMax spectrophotometer (Molecular Devices 197 Corporation) at $570 \mathrm{~nm}$ with the reference wavelength at $630 \mathrm{~nm}$.

199 Post-treatment THP1 cells were washed twice with RPMI and fixed for 45 min in a fixative solution 200 (2\% Glutaraldehyde in complete culture medium). The samples were centrifuged, and the pellet 201 fixed again with 1.5\% Glutaraldehyde solution in Na-Cacodylate buffer $0.1 \mathrm{M}$. A final post-fixation 202 (2 h) in $1 \% \mathrm{OsO}_{4}$ solution in Na-Cacodylate buffer $0.1 \mathrm{M}$ was performed. The samples were mixed 203 with uranyl acetate $2 \%(\mathrm{w} / \mathrm{v})$ and incubated for $5 \mathrm{~min}$, and then, $5 \mu \mathrm{l}$ was applied to carbon-coated 204 copper grids. Images were taken using a JEOL 2100 200-kV scanning and transmission electron 205 microscope with a Thermo Fisher Noran System 6 elemental analysis system. TEM was operated 206 at $80 \mathrm{kV}$, and images were obtained at 2,000× to 12,000× magnification. (Electron Microscopy 207 Facility, Iowa State University). 
Mitochondrial shape descriptors and size measurements were obtained using ImageJ (National Institutes of Health) by manually tracing only clearly discernible outlines of mitochondria on TEM micro-graphs (Picard et al., 2013). Surface area (or mitochondrial size) is reported in $\mu \mathrm{m}^{2}$; perimeter in $\mu \mathrm{m}$; circularity [4*(surface area/perimeter2)]; and Feret's diameter represents the longest distance $(\mu \mathrm{m})$ between any two points within a given mitochondrion. Computed values were imported into Microsoft Excel and Prism 8.0 for data analysis.

\section{Subcellular fractionation}

Whole cell and subcellular protein lysate extractions (cytosol and mitochondria) were performed at $4^{\circ} \mathrm{C}$ using cold reagents. For whole cell protein lysates, cell pellets were subjected to lysis using RIPA buffer [with protease and phosphatase inhibitors] (ThermoFisher Scientific). Subcellular fractionation of cell pellets for isolation of mitochondria was done using the Mitochondria Isolation Kit for Cultured Cells (ThermoFisher Scientific) according to the manufacturer's instructions. The whole cells, cytosolic fraction and isolated mitochondria were lysed with RIPA buffer [with protease and phosphatase inhibitors] for $30 \mathrm{~min}$ at $4^{\circ} \mathrm{C}$ and periodic sonication on ice, followed by centrifugation to collect lysate. Protein concentration of fractions were determined by Bradford assay (Bio-Rad) and were stored at $-80^{\circ} \mathrm{C}$ until use.

\section{Mt DNA isolation and long-range PCR}

To determine mitochondrial DNA (mtDNA) leakage, mtDNA was isolated from mitochondria-free cytosolic fraction of the cells. Mitochondrial DNA from cytosolic fractions was extracted using the Genomic DNA Purification kit (ThermoFisher Scientific) as per the manufacturer's instructions. The purity and concentration of the isolated DNA was measured using NanoDrop (NanoVue Plus Spectrophotometer, GE Healthcare). Due to low concentrations, the mtDNA was first amplified by long range PCR. The primers used were: mtDNA gene, sense: 5'TGAGGCCAAATATCATTCTGAGGGGC-3' and 5'TTTCATCATGCGGAGATGTTGGATGG-3' (Liu et al., 2015). PCR reactions were performed at $94^{\circ} \mathrm{C}$ for $1 \mathrm{~min}$ followed by 30 cycles at $98^{\circ} \mathrm{C}$ for $10 \mathrm{~s}, 60^{\circ} \mathrm{C}$ for $40 \mathrm{~s}, 68^{\circ} \mathrm{C}$ for $16 \mathrm{~min}$ and a final elongation for 10 min (Liu et al., 2015). Confirmation of the presence of mtDNA was done by separating the product by electrophoresis on a $0.8 \%$ agarose gel stained with ethidium bromide. The concentration of amplified mtDNA obtained was adjusted to ensure equal amounts of template mtDNA in each sample used for qPCR reaction.

\section{Quantitative Real-Time PCR}


240 Change in fold change of mtDNA was measured by qPCR with primers specific to mitochondrial 241 NADH dehydrogenase 1 (mtND1). $5 \mu \mathrm{L}$ of SYBR Green Mastermix (Thermo Fisher Scientific), 1

$242 \mu \mathrm{L}$ of primers, 2-3 $\mu \mathrm{L}$ of DNase/RNase free water and $1 \mu \mathrm{g}$ of amplified mtDNA was used. The 243 primers for genes of interest were synthesized at lowa State University's DNA Facility. The 244 primers used were: mtND1 gene, sense: 5'-GGCTATATACAACTACGCAAAGGC-3' and 245 antisense: 5'-GGTAGATGTGGCGGGTTTTAGG-3'; 16s (housekeeping gene), sense: 5'246 CCGCAAGGGAAAGATGAAAGAC-3' and anti-sense: 5'-TCGTTTGGTTTCGGGGTTTC-3'. No247 template and no-primer controls and dissociation curves were run for all reactions to exclude 248 cross-contamination. The qRT-PCR reactions were run in a QuantiStudio 3 system 249 (ThermoFisher) and the data was analyzed using $2^{-\triangle \Delta C T}$ method (Livak and Schmittgen, 2001). Western blot analysis

251 Lysates (whole cell, cytosol and MT) containing equal amounts of protein (20 $\mu \mathrm{g} / \mathrm{sample})$, along 252 with a molecular weight marker (Bio-Rad), were run on 10-15\% sodium dodecyl 253 sulfate/polyacrylamide gel electrophoresis (SDS-PAGE) as previously described(Bhat et al., 254 2019). Proteins were transferred to a nitrocellulose membrane and nonspecific binding sites were 255 blocked with Licor Odyssey blocking buffer. To investigate mitochondrial dysfunction, the 256 membranes were then incubated with different primary antibodies such as MFN1, MFN2, OPA1, 257 DRP1, PINK1, Parkin, BNIP3, Cytochrome C, COX4i2, Bcl-2, Bcl-XL, mtTFA, SOD2, Caspase 1 258 and Caspase 3 (1:1000 dilution). HMGB1 expression in mitochondrial fractions was measured 259 using anti-HMGB1 antibody (1:5000 dilution). Next, membranes were incubated with one of the 260 following secondary antibodies: Alexa Fluor 680 goat anti-mouse, Alexa Fluor 680 donkey anti261 rabbit or Alexa Fluor 800 donkey anti-rabbit (1:10,000; Invitrogen). To confirm equal protein 262 loading, blots were probed with a $\beta$-actin antibody (AbCam; 1:10,000 dilution). Western blot 263 images were captured using Odyssey ${ }^{\circledR}$ CLx IR imaging system (LI-COR Biotechnology) and 264 analysis was performed using ImageJ (National Institutes of Health).

266 Cells were seeded (50,000 cells/well) in a 96-well culture plate and treated for 24 hours. After 267 treatment, the media was removed and $100 \mu \mathrm{l}$ of $200 \mathrm{nM}$ MitoTracker green and $5 \mu \mathrm{M}$ MitoSOX 268 red dye diluted in 1\% FBS-containing RPMI medium was added into each well and incubated at $26937^{\circ} \mathrm{C}$ for $15 \mathrm{~min}$. Following incubation, the cells were washed with $1 \%$ FBS-containing RPMI 270 medium and fluorescence intensity was measured by spectrophotometer reading taken at 271 excitation/emission wavelengths of $485 / 520 \mathrm{~nm}$ and 510/580, respectively (SpectraMax M2 
272 Gemini Molecular Device Microplate Reader). The results were expressed as percentage mean

273 fluorescence intensity (\%MFI) relative to control.

274 Mitochondrial calcium influx measurement by rhod-2AM staining

275 Mitochondrial calcium influx $\left(\left[\mathrm{Ca}^{2+}\right]_{\text {mito }}\right)$ in THP1 cells was measured using the rhod-2AM dye. The 276 concentration of the isolated mitochondria was measured by Bradford assay in order to maintain 277 consistency in the number of mitochondria loaded into the wells of a 96-well plate. A protein 278 concentration of 100 ug was loaded into each well and10 $\mu$ M Rhod-2AM (Abcam) dye diluted in $2791 \%$ FBS-containing RPMI medium was added and incubated at $37^{\circ} \mathrm{C}$ for 30 minutes in order to 280 stain the mitochondria. The cells were washed with 1\% FBS-containing RPMI medium and 281 fluorescence was read at excitation/emission wavelengths of $552 \mathrm{~nm} / 581 \mathrm{~nm}$ using a 282 spectrophotometer reader (SpectraMax M2 Gemini Molecular Device Microplate Reader).

Griess assay

284 Griess assay was performed as described previously (Gordon et al., 2011). Briefly, nitric oxide 285 secretion was measured (representing reactive nitrogen species (RNS)) as nitrite levels in cell 286 culture media using Griess reagent (Sigma-Aldrich) and sodium nitrite standard curve, prepared 287 using a stock solution of $200 \mu \mathrm{M}$. The assay was performed in a 96 well-plate and absorbance 288 was measured at $550 \mathrm{~nm}$ (SpectraMax M2 Gemini Molecular Device Microplate Reader). The 289 results were expressed as $\mu \mathrm{M}$ concentration of nitrite secreted.

Statistical analysis

291 Data analysis and graphical representation was performed using GraphPad Prism 8.0 software 292 (GraphPad Prism 8.0, La Jolla, CA, USA). Data was analyzed with one-way ANOVA with Tukey's 293 multiple comparison test and a $p$-value of $<0.05$ was considered to be statistically significant.

294 Results

295 Exposure to ODE impacts the cellular and mitochondrial morphology

296 TEM images showed that THP1 cells treated with media alone (controls) showed normal 297 morphology with healthy mitochondria (Fig. 2a \& 2b). After ODE treatment, cytoplasmic 298 vacuolization and pseudopod formation was observed which suggests differentiation of the cells 299 to form activated macrophages (Fig. 2a) (Krysko et al., 2006). In addition, the mitochondria 300 seemed larger in size and some were elongated with reduced cristae number and/or deformed 301 cristae (Fig. 2b). On addition of $2.5 \mu \mathrm{M}$ ethyl pyruvate (EP), formation of multinucleated giant cells 
was observed, which is stated to be commonly observed in diverse infectious and non-infectious inflammatory conditions (Milde et al., 2015; Miron and Bosshardt, 2017). Similar to ODE exposure, the mitochondria were swollen and showed disorganized cristae, along with the presence of calcium sequestration bodies in the mitochondrial matrix (Fig. 2b). In contrast, exposure to $10 \mu \mathrm{M}$ mitapocyanin (MA) seemed to oppose the impact of ODE on the cells and restore it (Fig. 2b). Almost no cytoplasmic vacuolization was observed, and mitochondria showed decreased signs of damaged cristae, albeit conformed to an elongated morphology (Fig. 2a \& $2 b)$. This suggests that MA could have a protective effect on ODE exposed macrophages.

In order to quantify what was observed in the TEM images, mitochondria were individually traced from the TEM. Compared to controls, exposure to ODE significantly reduced the mitochondrial size (Fig. 2c). A similar decrease was seen in the presence of MA as well, whereas EP significantly increased the size, similar to that of control (Fig. 2c). A similar pattern was seen with the mitochondrial circularity, co-treatment with MA showing the most decrease compared to other treatments (Fig. 2d). Other morphological parameters such as perimeter, and Feret's diameter did not differ between any of the treatment conditions (Fig. 2e \& 2f). Taken together, this suggests that exposure to ODE activates a specific mechanism that acts towards altering the mitochondrial dynamics within the cell.

\section{Targeted antioxidant therapy promotes mitochondrial fission}

The mitochondrial membrane is remodeled continuously through cycles of fission and fusion events. The delicate balance of these events helps in controlling mitochondrial structure and function (Tilokani et al., 2018; Wai and Langer, 2016). In order to accurately interpret the impact of ODE exposure on the morphology of mitochondria, the expression of markers responsible for these dynamic events was observed. During ODE exposure, the expression of mitofusin 2 (MFN2) was significantly increased compared to control (Fig. 3a \& 3c). In contrast, mitofusin 1 (MFN1) and optic atrophy 1 (OPA1) did not show any significant changes in protein levels between control and treatments (Fig. 3a, 3b \& 3d). MFN1 and MFN2 are outer mitochondrial membrane GTPases that are responsible for the promotion of mitochondrial fusion (Tilokani et al., 2018). MFN2 alone can induce mitochondrial fragmentation and is a crucial regulator of mitochondria-endoplasmic reticulum (ER) contact site tethering (Filadi et al., 2018; Tilokani et al., 2018). Increased expression of dynamin-related protein 1 (DRP1) was observed in both ODE and MA treatments, indicative of mitochondrial fission as a result of oxidative stress and/or increased cytosolic calcium levels (Fig. 3e) (Eisner et al., 2018). Furthermore, to corroborate whether mitochondrial morphological changes are associated with mitochondrial number, change in mitochondrial mass 
on treatment was measured by mitotracker green fluorescence. There was a significant increase in the mitochondrial mass on exposure to ODE and EP, while on exposure to MA levels were comparable to controls (Fig. 3f). This could be a cellular response in order to compensate for the reduced mitochondrial function (Nugent et al., 2007). Collectively, the results suggest an effort to rescue mitochondrial biogenesis by increased MFN2 mediated fusion in response to ODEinduced cellular stress by maintaining a functional population of mitochondria within the cell.

\section{ODE exposure induces selective targeting of mitochondria for autophagy (mitophagy)}

Due to the increased MFN2 expression observed, it can be questioned whether this increase is favoring the process of mitochondrial elimination (Fig. 3a \& 3c). This process involved in the regulation of mitochondrial dynamics is also known to be closely associated with the process of mitochondrial quality control by autophagy, known as mitophagy (Ding and Yin, 2012; Filadi et al., 2018). Mitophagy is critical for maintaining proper cellular functions (Ding and Yin, 2012). Investigation of whether the mitochondria was subjected to autophagic clearance on ODE exposure was done. The expression of the two important mediators of mitophagy, PTEN-induced kinase 1 (PINK1) and the E3 ubiquitin protein ligase Parkin, were investigated. It was observed that there was increased Parkin expression in the presence of ODE compared to controls, while expression of PINK1 remained unchanged (Fig. 4a \& 4c). Expression of Parkin remained comparable to the control in the presence of EP or MA (Fig. $4 a \& 4 b$ ). Parkin has been shown to be highly essential in the induction of mitophagy (Ding and Yin, 2012; D. Narendra et al., 2010; Narendra et al., 2008; D. P. Narendra et al., 2010). PINK1 and Parkin are known to physically interact with each other in order to induce mitophagy, and the translocation of Parkin to the mitochondria is said to be dependent on PINK1 (Narendra et al., 2008; D. P. Narendra et al., 2010). This indicates that ODE-induced cellular stress is leading to Parkin mediated mitochondrial clearance.

The expression of BNIP3, a mitochondrial $\mathrm{Bcl}-2$ Homology $3(\mathrm{BH})$-only protein, was also observed. BNIP3 activates the mitochondrial permeability transition (MPT), which is associated with increased ROS production and excessive autophagy (Ney, 2015). BNIP3 levels remained unchanged on exposure to ODE and EP (Fig. 4a \& 4d). While, co-treatment with MA significantly decreased BNIP3 expression (Fig. 4d). This indicates that although the process of mitophagy may or may not be occurring via BNIP3, MA is certainly BNIP3 mediated MPT thus having a protective effect on the mitochondria.

\section{ODE exposure impacts mitochondrial membrane permeability}


Mitochondrial oxidative phosphorylation (OXPHOS) pathway is critical in determining and maintaining the immunomodulatory phenotype of activated macrophages (Kelly and O'Neill, 2015). Considering the changes in mitochondrial structure and dynamics, the question of whether ODE has an impact on the mitochondrial OXPHOS pathway was investigated. ODE increased levels of cytochrome $\mathrm{c}$ in the cytosol of the cells, compared to that in the mitochondrial fraction (Fig. 5a-5c). Release of cytochrome $\mathrm{c}$ is considered a key initial step in the apoptotic process (Cai et al., 1998; Ott et al., 2002). On the other hand, MA significantly decreased the levels of cytosolic cytochrome C (Fig. 5a-C). Concurrently, there is a significant decrease in the expression of lungspecific isoform of cytochrome c oxidase (COX4i2) in the mitochondrial fraction on ODE exposure, with no change on treatment with either EP or MA (Fig. $5 d$ ). COX4i2 is considered to be a ratelimiting step of the electron transport chain (ETC) in intact mammalian cells under physiological conditions (Hüttemann et al., 2012). A loss of expression would suggest dysfunctional OXPHOS pathway (Hüttemann et al., 2012). There is also an increase in superoxide dismutase 2 (SOD2) in the cytosol during ODE exposure (Fig. 5e). Treatment with EP or MA significantly decreased SOD2 expression compared to ODE (Fig. 5e). Presence of SOD2 is known to impart tolerance during high oxidative stress and reduce superoxide accumulation withing the mitochondria (Fukui and Zhu, 2010; Ishihara et al., 2015). To identify whether this is true, mitochondrial superoxide levels was measured by MitoSOX fluorescence. ODE significantly decreased the mitochondrial superoxide, while treatment with MA increased the levels comparable to control (Fig. 5f). ODE mediated decrease could either be a consequence of a leaky mitochondrial membrane or the action of high SOD2 expression. Reactive nitrite species (RNS) released into the extracellular environment was measured by griess assay. ODE exposure increased the levels of RNS in media at 24 hours, which treatment with EP and MA significantly attenuated RNS secretion (Fig. 5g). The results collectively show that in response to ODE, there is an increase in mitochondrial membrane permeability leading to leakage of core proteins involved in the maintenance of mitochondrial function. MA, on the other hand, seems to be maintaining the mitochondrial membrane integrity by acting as an inhibitor of peroxynitrite formation and RNS secretion, thus potentially restoring the damage induced on ODE exposure (Ghosh et al., 2016; Stefanska and Pawliczak, 2008).

\section{ODE induces the secretion of mitochondrial DAMPs}

Mitochondrial secondary messengers, which are mitochondrially derived molecules, can act as mitochondrial damage-associated molecular patterns (mtDAMPs) when produced excessively or are secreted into other cellular locations (Cloonan and Choi, 2012). These mtDAMPs result in the 
induction of a cascade of inflammatory responses withing the cell, thus resulting in adverse effects on the cell and tissue (Zhang et al., 2010). The levels of mitochondrial transcription factor A (mtTFA) measured in the mitochondrial and cytosolic fractions, showed a significant increase in mtTFA in both mitochondria and cytosol on exposure to ODE (Fig. 6a \& 6c). While MA treatment increased mtTFA levels in the mitochondria compared to ODE (Fig. 6a \& 6b). At normal physiological levels, mtTFA is an important regulator of mitochondrial DNA integrity, which when leaked out from mitochondria acts as a mtDAMP promoting inflammatory responses (Julian et al., 2013). Due to the increase in mitochondrial membrane permeability and dysfunction seen previously, levels of mtDNA leaking into the cytosol was determined. ODE increased cytosolic mtDNA levels, which is abrogated on treatment with EP or MA (Fig. 6d). In addition, there is an increase in calcium $\left(\mathrm{Ca}^{2+}\right)$ influx into the mitochondria on exposure to ODE, with no significant change in the presence of either MA or EP co-treatment compared to ODE (Fig. 6e). An increase in mitochondrial matrix $\mathrm{Ca}^{2+}$ levels has been shown to increase ATP production and is a trigger for cell death (Finkel et al., 2015). The expression of mitochondrial HMGB1 was determined, as presence of HMGB1 in the mitochondrial matrix is said to be critical in the regulation of mitochondrial function (Tang et al., 2011). Compared to control, there is a decrease in mitochondrial HMGB1 on exposure to ODE, which does not seem to be rescued in the presence of MA (Fig. 6f). Whereas on EP treatment, mitochondrial HMGB1 is significantly increased compared to both ODE and MA exposure (Fig. 6f). These findings indicate that with the significant impact ODE has on the mitochondrial quality control and biogenesis there is a release of mtDAMPs into the cytosol which could be leading to a cascade of inflammatory responses consequently causing cell death (Qi et al., 2015; Tang et al., 2011).

\section{Mitoapocynin does not intervene in ODE mediated caspase-1 upregulation}

As mentioned previously, release of mtDAMPs results in the activation of various inflammatory responses. It has been shown that release of mtDNA and mitochondrial reactive oxygen species (mROS) activates the NLRP3 inflammasome pathway (Gong et al., 2018). Upstream of NLRP3 activation, cleavage of pro-caspase 1 to caspase 1 is seen due to increased influx of calcium induced by leaky mitochondria (Murakami et al., 2012). Based on these evidences, changes in the expression of pro-caspase 1 and caspase 1 was measured. Compared to control, ODE and MA exposure increased expression of pro-caspase 1, EP maintained the expression comparable to control (Fig. 7a \& 7b). There was a significant increase expression of cleaved caspase 1 (p10) on ODE exposure and MA co-treatment, with the former inducing a higher expression than the latter (Fig. 7a \& 7c). Treatment with EP significantly decreased cleavage, which is consistent with 
the expression of pro-caspase 1. Expression of pro-caspase 3 and its cleaved product, an apoptosis executioner, was measured in order to determine if ODE is inducing a caspase 3 mediated apoptosis. Although ODE decreased the expression of pro-caspase 3, no significance in the levels was observed on exposure to treatments (Fig. $7 \mathrm{~d} \& 7 \mathrm{e}$ ). In addition, there was no caspase 3 cleavage product observed on exposure to any of the treatments (Fig. $7 \mathrm{~d}$ ). This suggests that ODE could be mediating a downstream inflammatory cascade via caspase 1 cleavage and activation, i.e. NLRP3 inflammasome activation and pro-IL-1 $\beta$ cleavage and release. This is not remedied by co-treatment with either MA or EP. Whereas, caspase 3 may not be a key mediator in ODE mediated inflammation.

\section{Mitoapocynin therapy does not inhibit ODE induced apoptosis}

The production of ROS is known to be a trigger for cell death(Brand et al., 2004; Kim, 2005). The antiapoptotic $\mathrm{Bcl}-2$ family proteins $\mathrm{Bcl}-2$ and $\mathrm{Bcl}-\mathrm{XL}$ play an important role in inhibiting mitochondria-dependent extrinsic and intrinsic cell death pathways (Green and Kroemer, 2004). To identify the impact OD-induced mitochondrial dysfunction and rescue may have on cellular apoptosis, expression of $\mathrm{Bcl}-2$ and $\mathrm{Bcl}-\mathrm{XL}$ were measured. ODE decreased the expression of $\mathrm{Bcl}-$ 2, with no change on MA or EP intervention (Fig. 8a). On the other hand, Bcl-XL expression was downregulated on ODE exposure, but was significantly increased on treatment with MA or EP (Fig. 8b). This change in expression of Bcl-XL was corroborated by measuring cell viability by MTT colorimetric assay. The percentage cell viability pattern observed correlated with the patter of expression of $\mathrm{Bcl}-\mathrm{XL}$, where loss of cell viability on OD exposure was rescued by treatment with EP or MA (Fig. 8c). Together, these results are indicative that the increase in $\mathrm{Bcl}-\mathrm{XL}$ expression on treatment with EP or MA, could be blocking the effect of BNIP3 (Fig. 4a \& 4d) in inducing the loss of mitochondrial membrane permeability or the activation of caspase dependent or independent apoptotic pathway (Kim, 2005). Thus, regulating the production of ROS and decreasing the probability of apoptotic and non-apoptotic cell death.

\section{Discussion}

Airway inflammation due to persistent exposure to OD is a key contributor to the development of respiratory symptoms and airflow obstruction in exposed workers (Cole et al., 2000; Nordgren and Charavaryamath, 2018). Continuous exposure to organic dust has been shown to alter innate immune responses in the airways (Charavaryamath and Singh, 2006; Sethi et al., 2017; Wunschel and Poole, 2016). These responses include cellular recruitment, release of pro-inflammatory cytokines and reactive species (ROS/RNS) (Bhat et al., 2019; Nath Neerukonda et al., 2018; 

Sahlander et al., 2012; Sethi et al., 2017). Previous studies have provided a direct link between such innate immune signaling and mitochondrial dynamics suggesting a crucial role in the activation and control of airway disease progression (Cloonan and Choi, 2012; Eisner et al., 2018). In vitro and in vivo studies have shown a link between airway diseases such as, influenza, sepsis-induced lung injury, pneumonia, and RSV infection, and facets of mitochondrial responses (Cloonan and Choi, 2016; Wunschel and Poole, 2016). In this study, using THP1 cells as an in vitro model for alveolar macrophages we provide evidence of significant changes in the dynamics, integrity and function of cellular mitochondria upon exposure to OD and how the use of mitoapocynin (MA), a novel mitochondrial targeting NOX2 inhibitor, or ethyl pyruvate (EP), an inhibitor of translocation of HMGB1, could rescue OD-induced mitochondrial changes and reduce inflammation.

Our TEM results demonstrate that, upon ODE exposure, there is increased presence of cytoplasmic vacuoles and pseudopods which is a characteristic feature of an activated macrophage ${ }^{28}$. Treatment with MA or EP did not prevent the ODE-induced morphological changes. When cells were exposed to EP co-treatment, we found formation of multinucleated giant cells (MGC). MGC are a common feature of granulomas that develop during certain infections, the most prominent example being tuberculosis or as a consequence of foreign body reactions (FBR) (Milde et al., 2015; Miron and Bosshardt, 2017).

To understand the impact of OD-induced inflammation on mitochondrial biogenesis, we explored factors involved in mitochondrial morphological changes. Mitochondria are highly dynamic organelles whose functions are essential for cell survival. They continuously change their function, position, and structure to meet the metabolic demands of the cells during homeostatic conditions as well as at times of stress (Eisner et al., 2018; Wai and Langer, 2016). With our TEM findings we observe distinct changes in the mitochondrial surface area and circularity on OD exposure and antioxidant therapy indicating that OD-exposure has an impact on mitochondrial dynamics and functions.

Mitochondria contain outer and inner mitochondrial membranes (OMM and IMM, respectively), which border the intermembrane space (IMS) and the matrix. Each of these compartments has discrete functions in metabolism, biosynthetic pathways, and signaling (Pagliarini and Rutter, 2013). Mitochondrial dynamics involve reshaping, rebuilding, and recycling events that support mitochondrial stability, abundance, distribution, and quality, and allow compensatory changes when cells are challenged (Mishra and Chan, 2014). Key mitochondrial reshaping mechanisms are mitochondrial fission and fusion. Mitochondrial fission is characterized by division of one 
mitochondrion into two daughter mitochondria, whereas mitochondrial fusion is the union of two mitochondria resulting in one mitochondrion(Mishra and Chan, 2014). The deregulation of these spatio-temporal events results in either a fragmented network characterized by a large number of small round-shaped mitochondria or a hyper fused network with elongated and highly connected mitochondria (Tilokani et al., 2018; Wai and Langer, 2016). These balanced dynamic transitions are not only required to ensure mitochondrial function but also to respond to cellular needs by adapting to nutrient availability and metabolic state of the cell.

Mitochondrial fusion is mediated by dynamin-related GTPases mitofusin 1 and 2 (MFN1/2) on the outer mitochondrial membrane (OMM) and by dynamin-related protein optic atrophy 1 (OPA1) on the inner mitochondrial membrane (IMM). Mitochondrial fission requires the recruitment of dynamin-related protein 1 (DRP1) from the cytosol to its specific receptors (Mishra and Chan, 2014; Tilokani et al., 2018). Lack of either MFN1 or MFN2 expression can display aberrant mitochondrial morphology. While a lack of MFN1 induces mitochondrial fragmentation, the absence of MFN2 exhibits swollen spherical mitochondria. In our findings, we see increased MFN2 expression on OD exposure, which could be leading to increased mitochondrial fragmentation and increased mitochondrial mass (Chen et al., 2003). An increase in the rate of mitochondrial proliferation is probably a cellular response to counteract the loss of mitochondrial function and recover ATP synthesis capacity. We also see an increase in DRP1 expression upon exposure to both ODE and MA co-treatment. Mitochondrial fission is essential for the inheritance and partitioning of mitochondria during cell division. Inhibition of DRP1-mediated mitochondrial fission has been reported to cause cellular dysfunction and replication (Qi et al., 2015). This can be corroborated by the decrease in cell viability with ODE exposure. The low mitochondrial mass observed with exposure to MA could be a means by which the antioxidant therapy is overcoming the increase in dysfunctional mitochondria.

Studies have shown that the MFN2 mediates Parkin, an E3 ubiquitin ligase, recruitment to damaged mitochondria (Filadi et al., 2018). Parkin binds to MFN2 in a PINK1-dependent manner and promotes Parkin-mediated ubiquitination of damaged mitochondria, thus leading to a mitochondria quality control process, known as mitophagy (Ding and Yin, 2012; Narendra et al., 2008; D. P. Narendra et al., 2010). This corroborates our finding wherein we see an increase in MFN2 and Parkin expression upon ODE exposure, from which we can conclude that OD-induced cell stress leads to mitophagy. Mitophagy is a process of mitochondrial quality control where damaged or defective mitochondria are removed by selective encapsulation into doublemembraned autophagosomes that are delivered to lysosome for degradation (Ding and Yin, 
531 2012). Mitochondrial biogenesis and mitophagy allow cells to quickly replace metabolically 532 dysfunctional mitochondria.

533 Albeit no significant change in BNIP3 expression was observed with exposure to OD or EP. 534 However, BNIP3 expression was decreased on intervention with MA. This brings into question 535 how the low levels of BNIP3 is affecting the mitochondrial and cellular function. BNIP3, a 536 transmembrane protein located in the OMM, imparts some pro-cell death activity and is known to 537 regulate mitophagy (Ney, 2015). BNIP3 has been shown to activate the mitochondrial 538 permeability transition (MPT) and degradation of proteins involved in oxidative phosphorylation, 539 in turn leading to cell death without cytochrome c release or caspase activation (Landes et al., 2010; Quinsay et al., 2010; Rikka et al., 2011; Velde et al., 2000). On addition of BNIP3 to isolated mitochondria, it was observed that BNIP3 caused cytochrome c release, depolarization, and swelling (Kim et al., 2002). This phenomenon has been linked to BNIP3-mediated permeabilization of inner and outer mitochondrial membrane involving the disruption of OPA1 complex and remodeling of the inner mitochondrial membrane (Landes et al., 2010). In comparison to our findings, we can assume that MA-induced decrease in BNIP3 expression could be reducing MPT and cell death, thus improving overall cellular function. Another potential mechanism by which BNIP3 is promoting apoptosis is by competition for binding to $\mathrm{Bcl}-2$ (or a related protein) which liberates Beclin-1 from Bcl-2 complexes and activates autophagy. There is evidence showing that Bcl-XL enhances BNIP3-induced mitophagy (Maiuri et al., 2007; Pattingre et al., 2005). This correlates with our findings wherein we see decreased $\mathrm{Bcl}-\mathrm{XL}$ expression on exposure to OD which is significantly upregulated on intervention with EP or MA. Taken together we see a decrease in overall cell viability on OD exposure which is rescued by MA intervention.

553 One of the prominent players in cell death is cytochrome c. Based on previous studies, we 554 expected that with the decrease in cell viability and induction of mitophagy there would be a 555 release of cytochrome $\mathrm{c}$ from the mitochondria into the cytosol. Cytochrome $\mathrm{c}$, a peripheral protein 556 of the mitochondrial inner membrane (IMM), is known to function as an electron shuttle between 557 complex III and complex IV of the respiratory chain and its activity (Cai et al., 1998; Garrido et al., 558 2006). And its release from the IMM has been implicated in caspase activation and mitochondrial 559 outer membrane permeabilization (MOMP), leading to cell death. Cumulative data suggest that cytochrome c release does not always take place in an all-or-nothing manner as previously 561 believed, but instead follows a biphasic kinetics (Ott et al., 2002). The first wave is induced by 562 apoptotic signals directed to mitochondria which provokes MOMP and cytochrome c release, thus 563 disrupting the electron transport and leading to an increased generation of ROS. The second 
564

565

566

567

568

569

570

571

572

573

574

575

576

577

578

579

580

581

582

583

584

585

586

587

588

589

590

591

592

593

594

595

596

597 wave involves cytochrome c mediated activation of caspases that subsequently enters the mitochondria through the permeabilized OMM and induce the complete block of the respiratory chain, eventually resulting in cell death. Comparing this to our findings we observe that with the increase in cytosolic cytochrome c on OD exposure there is a deficiency of levels of COX4i2 (COX subunit 4 isoform 2), a terminal enzyme in the OXPHOS machinery. Loss of COX4i2 results in decreased COX activity and decreased ATP levels (Hüttemann et al., 2012). This loss is not reversed by the use of antioxidant therapy, albeit MA was capable of downregulating the release of cytochrome c. This is indicative that although antioxidant therapy can decrease the cytosolic release of cytochrome $c$, there could be other secondary factors resulting in the loss of COX4i2. NADPH oxidase is the main source of ROS that is closely linked to mitochondrial ROS production (Zorov et al., 2014). Growing evidence suggests that ROS generated can increase expression of proinflammatory mediators (Bhat et al., 2019; Brand et al., 2004). Indeed, various PAMP molecules can stimulate ROS production by NADPH oxidase, especially NOX1, NOX2, NOX4 (Ghosh et al., 2016). Being a NOX2 inhibitor, treatment with MA seems to bring mitochondrial superoxide levels to that of the controls, whereas with OD and EP we see a decrease. This could be a consequence of a leaky mitochondria which is enabling the release of the superoxide ion into the cytosol thus promoting further damage to the cell. On the other hand, seeing the increase in the SOD2 expression allows us to believe that there are factors promoting the attenuation of oxidative stress mediated cellular injury. This increase could be due to a variety of proinflammatory cytokines, such as interleukin 1 (IL-1), IL-4, IL-6, tumor necrosis factor $\alpha$, interferon $\mathrm{\gamma}$, and the bacterial endotoxin lipopolysaccharide, which are considered to be robust SOD2 activators (Fukui and Zhu, 2010, p. 2). SOD2 is also said to be regulated by RNS, where increased peroxynitrite levels can lead to its enzymatic inhibition (Redondo-Horcajo et al., 2010). These antagonistic roles that peroxynitrite and superoxide radicals have in regulating SOD2 expression and activity leads us to believe that mitochondrial antioxidant response is dysregulated.

A wide variety of mitochondrial-derived molecules, which act as second messengers, can also behave as mitochondrial damage-associated molecular patterns (mtDAMPs) when produced in excess or released into an alternative cellular compartment. Activation of MPT during mitochondrial dysfunction has also been shown to cause leakage of mtDAMPs, primarily mitochondrial DNA (mtDNA) into the cytosol and activating caspase 1 (Nakahira et al., 2011). The release of mtDNA has been shown to cause neutrophil mediated organ injury by systemic inflammatory reaction via the activation of DNA sensor cyclic GMP-AMP synthase (cGAS) and TLR9 pathway, intracellularly (West et al., 2015; Zhang et al., 2010). Mitochondrial transcription 
598 factor $A$ (mtTFA) is an integral regulator of mtDNA integrity, which, when released from 599 mitochondria, acts as a mtDAMP to regulate inflammatory responses (Julian et al., 2013). 600 Release of mtTFA along with mtDNA during cell damage amplified TNFa and type 1 interferon 601 release, which plays a critical role in promoting sterile inflammation and autoimmune diseases 602 (Cantaert et al., 2010; CHAUNG et al., 2012; Julian et al., 2012). This is in line with our findings 603 where we see an increase in the cytosolic release of mtTFA and mtDNA on OD exposure. 604 Although antioxidant therapy did not have a significant impact on reducing release of mtTFA, it 605 did however decrease the release of mtDNA. Being a homolog of mtTFA, HMGB1 was 606 investigated to understand the impact of its translocation into the mitochondrial can have (Parisi 607 and Clayton, 1991). Under pathophysiological conditions, nuclear HMGB1 is immediately 608 transported to the cytoplasm and released into the extracellular space where it acts as a signaling 609 molecule regulating a wide range of inflammatory responses by binding to TLR2/4 and/or receptor 610 for advanced glycan end products (RAGE) (Bhat et al., 2019). It has been reported that HMGB1 611 rescues the impairment of mitochondrial function. In endothelial cells, the translocation of 612 endogenous HMGB1 from the nucleus to the mitochondria promotes mitochondrial reorganization 613 (Hyun et al., 2016; Stumbo et al., 2008). In cancer cells, exogenous HMGB1 enters the 614 mitochondria, which is followed by the formation of giant mitochondria (Gdynia et al., 2016; Hyun 615 et al., 2016). Therefore, it is likely that the nuclear HMGB1 export would be involved in aberrant 616 mitochondrial fission or the compensatory responses for maintenance of mitochondrial functions. 617 However, in the present study we see that treatment with MA does not revert back the levels of 618 HMGB1 within the mitochondria and match the levels observed on OD exposure.

619 Mitochondria are also key regulators calcium $\left(\mathrm{Ca}^{2+}\right)$ which control a diverse range of cellular 620 processes, including ROS production. $\mathrm{Ca}^{2+}$ influx into the mitochondrial matrix ([Ca $\left.\left.{ }^{2+}\right]_{\text {mito }}\right)$ has 621 been shown to be an important regulator of mitochondrial metabolism, and the mobilization of and 622 regulation of mitochondrial $\mathrm{Ca}^{2+}$ uptake has been linked to $\mathrm{Bcl}-\mathrm{XL}$ and voltage-dependent anion 623 channel (VDAC) interactions (Huang et al., 2013; Jouaville et al., 1999; Pitter et al., 2002). Any 624 aberrant increase in cytosolic $\mathrm{Ca}^{2+}$ and resultant $\left[\mathrm{Ca}^{2+}\right]_{\text {mito }}$ overload can be a trigger for cell death 625 (Finkel et al., 2015). This overload has also been linked to induction of MPT, resulting in 626 mitochondrial permeabilization (Finkel et al., 2015; Hunter et al., 1976). This is corroborated by 627 our findings where we see increase in expression of MPT-inducing markers and [Ca $\left.{ }^{2+}\right]_{\text {mito }}$ levels 628 on OD exposure. However, on our observation that $\mathrm{Bcl}-\mathrm{XL}$ is abrogated, it is safe to assume that 629 the $\mathrm{Ca}^{2+}$ influx could possibly be occurring via the interaction of VDAC with $\mathrm{Mcl}-1$, a Bcl-2 family 630 protein (Huang et al., 2014). $\mathrm{Ca}^{2+}$ signaling also plays a critical role in the activation of NLRP3 631 inflammasome by multiple stimuli (Murakami et al., 2012). This is corroborated by our findings 
632 wherein we see caspase-1 processing with the increase $\mathrm{Ca}^{2+}$ levels on OD exposure (Yu et al.,

633 2014). This would in turn lead to IL-1 $\beta$ processing and release into the extracellular space. The

634 use of MA does not seem to have any impact on the levels of $\mathrm{Ca}^{2+}$ accumulation within the

635 mitochondria which could be inducing an inflammatory cascade not mediated by mitochondria.

\section{Conclusion}

637 In conclusion, we document that co-treatment with EP and MA are partially protective as they 638 rescue some of the ODE-exposure induced deficits. However, these findings lead to new 639 mechanistic questions on how OD may be inducing mitochondrial dysfunction and cell death. OD 640 being a complex mixture of contaminants could be inducing a multifactorial immune response and 641 the mechanism underlying these responses are not yet well understood. Specific signatures of 642 mitochondrial dysfunction that are associated with disease pathogenesis and/or progression are 643 becoming increasingly important. Although our current study is limited with the use of a single 644 immortalized cell line as a model, it provides data on the impact of OD on mitochondrial biogenesis 645 and function. Future studies using functional (primary alveolar macrophages, precision cut lung 646 slices) and mouse model would be highly valuable.

\section{Acknowledgements}

648 We would like to thank Tracey Stewart at ISU's Roy J. Carver High Resolution Microscopy Facility 649 for assistance with transmission electron microscopy.

\section{Funding}

651 C.C. laboratory is funded through startup grant through lowa State University and a pilot grant (5 652 U54 OH007548) from CDC-NIOSH (Centers for Disease Control and Prevention-The National 653 Institute for Occupational Safety and Health). A.G.K. laboratory is supported by National Institutes 654 of Health grants (ES026892, ES027245 and NS100090).

\section{Potential Conflicts of Interest}

656 AGK has an equity interest in PK Biosciences Corporation located in Ames, IA.

657 The terms of this arrangement have been reviewed and approved by lowa State University per 658 its conflict of interest policies. All other authors have declared no potential conflicts of interest.

\section{Author contributions}

660 S.M. Bhat participated in the design of experiments, performed the experiments, analyzed the 661 data, and wrote the manuscript. D. Shrestha performed the calcium influx assay. N. Massey 
662

663

664

665

666

667

668

669

670

671

672

673

674

675

676

677

678

679

680

681

682

683

684

685

686

687

688

689

690

691

692

693

performed organic dust extraction. L. Karriker collected the organic dust samples and edited the manuscript. A.G. Kanthasamy provided mitoapocynin and edited the manuscript. C. Charavaryamath conceptualized the study, participated in the design of the experiments, performed dust extraction, participated in the interpretation of data and edited the manuscript. All authors have read and approved the final manuscript.

\section{Abbreviations}

OD: Organic Dust; ODE: Orgaic Dust Extract; EP: Ethyl Pyruvate; MA: Mitoapocynin; LPS: Lipopolysaccharide; PGN: Peptidoglycan; PAMPs: Pathogen Associated Molecular Patterns; COPD: Chronic Obstructive Pulmonary Disease; AHR: Airway hyperresponsiveness; ROS: Reactive Oxygen Species; RNS: Reactive Nitrogen Species; ATP: Adenosine Triphosphate; OXPHOS: Oxidative Phosphorylation; HMGB1: High Mobility Group Box 1; STAT: Signal Transducer and Activator of Transcription; TPP: Triphenylphosphonium; MPTP: 1-Methyl-4Phenyl-1,2,3,6-Tetrahydropyridine; iNOS: inducible Nitric Oxide Synthase; NOX: NADPH Oxidase; MTT: 3-[4,5-dimethylthiazole-2-yl]-2,5-diphenyltetrazolium bromide; TEM: Transmission Electron Microscopy; DMSO: Dimethyl Sufoxide; mtND1: mitochondrial NADH dehydrogenase 1; MFN: Mitofusin; OPA1: Optic Atrophy 1; DRP1: Dynamin-related protein 1; ER: Endoplasmic Reticulum; PINK1: PTEN- induced kinase 1; BNIP3: Bcl-2 Homology 3 (BH3)-only; MPT: Mitochondrial Permeability Transition; COX4i2: Cytochrome C Oxidase subunit 4 isoform 2; ETC: Electron Transport Chain; SOD2: Superoxide Dismutase 2; mtDAMPs: mitochondrial Damage Associated Molecular Patters; mtTFA: mitochondrial Transcription Factor A; MGC: Multinucleated Giant Cell; FBR: Foreign Body Reactions; OMM: Outer Mitochondrial Membrane; IMM: Inner Mitochondrial Membrane; IMS: Intermembrane Space; IL: Interleukin; cGAS: cyclic GMP-AMP synthase; TLR: Toll-like receptor; RAGE: Receptor for advanced glycation end products; VDAC: Voltage-dependent anion channel

\section{References}

1. Bhat, S.M., Massey, N., Karriker, L.A., Singh, B., Charavaryamath, C., 2019. Ethyl pyruvate reduces organic dust-induced airway inflammation by targeting HMGB1-RAGE signaling. Respiratory Research 20, 27. https://doi.org/10.1186/s12931-019-0992-3

2. Brand, M.D., Affourtit, C., Esteves, T.C., Green, K., Lambert, A.J., Miwa, S., Pakay, J.L., Parker, N., 2004. Mitochondrial superoxide: production, biological effects, and activation of uncoupling proteins. Free Radical Biology and Medicine 37, 755-767. https://doi.org/10.1016/j.freeradbiomed.2004.05.034 
3. Cai, J., Yang, J., Jones, DeanP., 1998. Mitochondrial control of apoptosis: the role of cytochrome c. Biochimica et Biophysica Acta (BBA) - Bioenergetics 1366, 139-149. https://doi.org/10.1016/S0005-2728(98)00109-1

4. Cantaert, T., Baeten, D., Tak, P.P., van Baarsen, L.G., 2010. Type I IFN and TNFa crossregulation in immune-mediated inflammatory disease: basic concepts and clinical relevance. Arthritis Research \& Therapy 12, 219. https://doi.org/10.1186/ar3150

5. Charavaryamath, C., Janardhan, K.S., Townsend, H.G., Willson, P., Singh, B., 2005. Multiple exposures to swine barn air induce lung inflammation and airway hyper-responsiveness. Respiratory Research 6, 50. https://doi.org/10.1186/1465-9921-6-50

6. Charavaryamath, C., Singh, B., 2006. Pulmonary effects of exposure to pig barn air. J Occup Med Toxicol 1, 10. https://doi.org/10.1186/1745-6673-1-10

7. CHAUNG, W.W., WU, R., JI, Y., DONG, W., WANG, P., 2012. Mitochondrial transcription factor A is a proinflammatory mediator in hemorrhagic shock. Int J Mol Med 30, 199-203. https://doi.org/10.3892/ijmm.2012.959

8. Chen, H., Detmer, S.A., Ewald, A.J., Griffin, E.E., Fraser, S.E., Chan, D.C., 2003. Mitofusins Mfn1 and Mfn2 coordinately regulate mitochondrial fusion and are essential for embryonic development. J Cell Biol 160, 189-200. https://doi.org/10.1083/jcb.200211046

9. Cloonan, S.M., Choi, A.M., 2012. Mitochondria: commanders of innate immunity and disease? Current Opinion in Immunology, Innate immunity / Antigen processing 24, 32-40. https://doi.org/10.1016/j.coi.2011.11.001

10. Cloonan, S.M., Choi, A.M.K., 2016. Mitochondria in lung disease. J Clin Invest 126, 809-820. https://doi.org/10.1172/JCl81113

11. Cole, D., Todd, L., Wing, S., 2000. Concentrated swine feeding operations and public health: a review of occupational and community health effects. Environ Health Perspect 108, 685699.

12. Ding, W.-X., Yin, X.-M., 2012. Mitophagy: mechanisms, pathophysiological roles, and analysis. Biol Chem 393, 547-564. https://doi.org/10.1515/hsz-2012-0119

13. Eisner, V., Picard, M., Hajnóczky, G., 2018. Mitochondrial dynamics in adaptive and maladaptive cellular stress responses. Nature Cell Biology 20, 755-765. https://doi.org/10.1038/s41556-018-0133-0

14. Filadi, R., Pendin, D., Pizzo, P., 2018. Mitofusin 2: from functions to disease. Cell Death Dis 9, 330. https://doi.org/10.1038/s41419-017-0023-6 
15. Finkel, T., Menazza, S., Holmström, K.M., Parks, R.J., Liu, Julia, Sun, J., Liu, Jie, Pan, X., Murphy, E., 2015. The Ins and Outs of Mitochondrial Calcium. Circ Res 116, 1810-1819. https://doi.org/10.1161/CIRCRESAHA.116.305484

16. Fukui, M., Zhu, B.T., 2010. Mitochondrial Superoxide Dismutase SOD2, but not Cytosolic SOD1, Plays a Critical Role in Protection against Glutamate-Induced Oxidative Stress and Cell Death in HT22 Neuronal Cells. Free Radic Biol Med 48, 821-830. https://doi.org/10.1016/j.freeradbiomed.2009.12.024

17. Garrido, C., Galluzzi, L., Brunet, M., Puig, P.E., Didelot, C., Kroemer, G., 2006. Mechanisms of cytochrome c release from mitochondria. Cell Death \& Differentiation 13, 1423-1433. https://doi.org/10.1038/sj.cdd.4401950

18. Gdynia, G., Sauer, S.W., Kopitz, J., Fuchs, D., Duglova, K., Ruppert, T., Miller, M., Pahl, J., Cerwenka, A., Enders, M., Mairbäurl, H., Kamiński, M.M., Penzel, R., Zhang, C., Fuller, J.C., Wade, R.C., Benner, A., Chang-Claude, J., Brenner, H., Hoffmeister, M., Zentgraf, H., Schirmacher, P., Roth, W., 2016. The HMGB1 protein induces a metabolic type of tumour cell death by blocking aerobic respiration. Nature Communications 7, 10764. https://doi.org/10.1038/ncomms10764

19. Ghosh, A., Langley, M.R., Harischandra, D.S., Neal, M.L., Jin, H., Anantharam, V., Joseph, J., Brenza, T., Narasimhan, B., Kanthasamy, A., Kalyanaraman, B., Kanthasamy, A.G., 2016. Mitoapocynin Treatment Protects Against Neuroinflammation and Dopaminergic Neurodegeneration in a Preclinical Animal Model of Parkinson's Disease. J Neuroimmune Pharmacol 11, 259-278. https://doi.org/10.1007/s11481-016-9650-4

20. Gong, Z., Pan, J., Shen, Q., Li, M., Peng, Y., 2018. Mitochondrial dysfunction induces NLRP3 inflammasome activation during cerebral ischemia/reperfusion injury. Journal of Neuroinflammation 15, 242. https://doi.org/10.1186/s12974-018-1282-6

21. Gordon, R., Hogan, C.E., Neal, M.L., Anantharam, V., Kanthasamy, A.G., Kanthasamy, A., 2011. A simple magnetic separation method for high-yield isolation of pure primary microglia. J. Neurosci. Methods 194, 287-296. https://doi.org/10.1016/j.jneumeth.2010.11.001

22. Green, D.R., Kroemer, G., 2004. The Pathophysiology of Mitochondrial Cell Death. Science 305, 626-629. https://doi.org/10.1126/science.1099320

23. Huang, H., Hu, X., Eno, C.O., Zhao, G., Li, C., White, C., 2013. An Interaction between Bcl$\mathrm{XL}$ and the Voltage-dependent Anion Channel (VDAC) Promotes Mitochondrial Ca2+ Uptake. J. Biol. Chem. 288, 19870-19881. https://doi.org/10.1074/jbc.M112.448290

24. Huang, H., Shah, K., Bradbury, N.A., Li, C., White, C., 2014. Mcl-1 promotes lung cancer cell migration by directly interacting with VDAC to increase mitochondrial $\mathrm{Ca} 2+$ uptake and 
reactive oxygen species generation. Cell Death \& Disease 5, e1482-e1482. https://doi.org/10.1038/cddis.2014.419

25. Hunter, D.R., Haworth, R.A., Southard, J.H., 1976. Relationship between configuration, function, and permeability in calcium-treated mitochondria. J. Biol. Chem. 251, 5069-5077.

26. Hüttemann, M., Lee, I., Gao, X., Pecina, P., Pecinova, A., Liu, J., Aras, S., Sommer, N., Sanderson, T.H., Tost, M., Neff, F., Aguilar-Pimentel, J.A., Becker, L., Naton, B., Rathkolb, B., Rozman, J., Favor, J., Hans, W., Prehn, C., Puk, O., Schrewe, A., Sun, M., Höfler, H., Adamski, J., Bekeredjian, R., Graw, J., Adler, T., Busch, D.H., Klingenspor, M., Klopstock, T., Ollert, M., Wolf, E., Fuchs, H., Gailus-Durner, V., Angelis, M.H. de, Weissmann, N., Doan, J.W., Bassett, D.J.P., Grossman, L.I., 2012. Cytochrome c oxidase subunit 4 isoform 2knockout mice show reduced enzyme activity, airway hyporeactivity, and lung pathology. The FASEB Journal 26, 3916-3930. https://doi.org/10.1096/fj.11-203273

27. Hyun, H.-W., Ko, A.-R., Kang, T.-C., 2016. Mitochondrial Translocation of High Mobility Group Box 1 Facilitates LIM Kinase 2-Mediated Programmed Necrotic Neuronal Death. Front Cell Neurosci 10. https://doi.org/10.3389/fncel.2016.00099

28. Ishihara, Y., Takemoto, T., Itoh, K., Ishida, A., Yamazaki, T., 2015. Dual Role of Superoxide Dismutase 2 Induced in Activated Microglia OXIDATIVE STRESS TOLERANCE AND CONVERGENCE OF INFLAMMATORY RESPONSES. J. Biol. Chem. 290, 22805-22817. https://doi.org/10.1074/jbc.M115.659151

29. Jouaville, L.S., Pinton, P., Bastianutto, C., Rutter, G.A., Rizzuto, R., 1999. Regulation of mitochondrial ATP synthesis by calcium: Evidence for a long-term metabolic priming. PNAS 96, 13807-13812. https://doi.org/10.1073/pnas.96.24.13807

30. Julian, M.W., Shao, G., Bao, S., Knoell, D.L., Papenfuss, T.L., VanGundy, Z.C., Crouser, E.D., 2012. Mitochondrial Transcription Factor A Serves as a Danger Signal by Augmenting Plasmacytoid Dendritic Cell Responses to DNA. The Journal of Immunology 189, 433-443. https://doi.org/10.4049/jimmunol.1101375

31. Julian, M.W., Shao, G., Vangundy, Z.C., Papenfuss, T.L., Crouser, E.D., 2013. Mitochondrial transcription factor $A$, an endogenous danger signal, promotes TNFa release via RAGE- and TLR9-responsive plasmacytoid dendritic cells. PLoS ONE 8, e72354. https://doi.org/10.1371/journal.pone.0072354

32. Kelly, B., O'Neill, L.A., 2015. Metabolic reprogramming in macrophages and dendritic cells in innate immunity. Cell Research 25, 771-784. https://doi.org/10.1038/cr.2015.68

33. Kim, H.S., Cho, I.H., Kim, J.E., Shin, Y.J., Jeon, J.-H., Kim, Y., Yang, Y.M., Lee, K.-H., Lee, J.W., Lee, W.-J., Ye, S.-K., Chung, M.-H., 2008. Ethyl pyruvate has an anti-inflammatory effect 
by inhibiting ROS-dependent STAT signaling in activated microglia. Free Radic. Biol. Med. 45, 950-963. https://doi.org/10.1016/j.freeradbiomed.2008.06.009

34. Kim, J.-Y., Cho, J.-J., Ha, J., Park, J.-H., 2002. The Carboxy Terminal C-Tail of BNip3 Is Crucial in Induction of Mitochondrial Permeability Transition in Isolated Mitochondria. Archives of Biochemistry and Biophysics 398, 147-152. https://doi.org/10.1006/abbi.2001.2673

35. Kim, R., 2005. Unknotting the roles of Bcl-2 and Bcl-xL in cell death. Biochemical and Biophysical Research Communications 336-343. https://doi.org/10.1016/j.bbrc.2005.04.161

36. Krysko, D.V., Denecker, G., Festjens, N., Gabriels, S., Parthoens, E., D'Herde, K., Vandenabeele, P., 2006. Macrophages use different internalization mechanisms to clear apoptotic and necrotic cells. Cell Death \& Differentiation 13, 2011-2022. https://doi.org/10.1038/sj.cdd.4401900

37. Landes, T., Emorine, L.J., Courilleau, D., Rojo, M., Belenguer, P., Arnauné-Pelloquin, L., 2010. The BH3-only Bnip3 binds to the dynamin Opa1 to promote mitochondrial fragmentation and apoptosis by distinct mechanisms. EMBO reports 11, 459-465. https://doi.org/10.1038/embor.2010.50

38. Langley, M., Ghosh, A., Charli, A., Sarkar, S., Ay, M., Luo, J., Zielonka, J., Brenza, T., Bennett, B., Jin, H., Ghaisas, S., Schlichtmann, B., Kim, D., Anantharam, V., Kanthasamy, A., Narasimhan, B., Kalyanaraman, B., Kanthasamy, A.G., 2017. Mito-Apocynin Prevents Mitochondrial Dysfunction, Microglial Activation, Oxidative Damage, and Progressive Neurodegeneration in MitoPark Transgenic Mice. Antioxidants \& Redox Signaling 27, 10481066. https://doi.org/10.1089/ars.2016.6905

39. Latchoumycandane, C., Anantharam, V., Kitazawa, M., Yang, Y., Kanthasamy, A., Kanthasamy, A.G., 2005. Protein kinase Cdelta is a key downstream mediator of manganeseinduced apoptosis in dopaminergic neuronal cells. J. Pharmacol. Exp. Ther. 313, 46-55. https://doi.org/10.1124/jpet.104.078469

40. Liu, J., Fang, H., Chi, Z., Wu, Z., Wei, D., Mo, D., Niu, K., Balajee, A.S., Hei, T.K., Nie, L., Zhao, Y., 2015. XPD localizes in mitochondria and protects the mitochondrial genome from oxidative DNA damage. Nucleic Acids Res 43, 5476-5488. https://doi.org/10.1093/nar/gkv472

41. Livak, K.J., Schmittgen, T.D., 2001. Analysis of Relative Gene Expression Data Using RealTime Quantitative PCR and the 2- $\Delta \Delta C T$ Method. Methods 25, 402-408. https://doi.org/10.1006/meth.2001.1262 
42. Maiuri, M.C., Le Toumelin, G., Criollo, A., Rain, J.-C., Gautier, F., Juin, P., Tasdemir, E., Pierron, G., Troulinaki, K., Tavernarakis, N., Hickman, J.A., Geneste, O., Kroemer, G., 2007. Functional and physical interaction between Bcl-XL and a BH3-like domain in Beclin-1. The EMBO Journal 26, 2527-2539. https://doi.org/10.1038/sj.emboj.7601689

43. Milde, R., Ritter, J., Tennent, G.A., Loesch, A., Martinez, F.O., Gordon, S., Pepys, M.B., Verschoor, A., Helming, L., 2015. Multinucleated Giant Cells Are Specialized for ComplementMediated Phagocytosis and Large Target Destruction. Cell Rep 13, 1937-1948. https://doi.org/10.1016/j.celrep.2015.10.065

44. Miron, R.J., Bosshardt, D.D., 2017. Multinucleated Giant Cells: Good Guys or Bad Guys? Tissue Engineering Part B: Reviews 24, 53-65. https://doi.org/10.1089/ten.teb.2017.0242

45. Mishra, P., Chan, D.C., 2014. Mitochondrial dynamics and inheritance during cell division, development and disease. Nature Reviews Molecular Cell Biology 15, 634-646. https://doi.org/10.1038/nrm3877

46. Murakami, T., Ockinger, J., Yu, J., Byles, V., McColl, A., Hofer, A.M., Horng, T., 2012. Critical role for calcium mobilization in activation of the NLRP3 inflammasome. Proc. Natl. Acad. Sci. U.S.A. 109, 11282-11287. https://doi.org/10.1073/pnas.1117765109

47. Nakahira, K., Haspel, J.A., Rathinam, V.A.K., Lee, S.-J., Dolinay, T., Lam, H.C., Englert, J.A., Rabinovitch, M., Cernadas, M., Kim, H.P., Fitzgerald, K.A., Ryter, S.W., Choi, A.M.K., 2011. Autophagy proteins regulate innate immune responses by inhibiting the release of mitochondrial DNA mediated by the NALP3 inflammasome. Nature Immunology 12, 222-230. https://doi.org/10.1038/ni.1980

48. Narendra, D., Kane, L.A., Hauser, D.N., Fearnley, I.M., Youle, R.J., 2010. p62/SQSTM1 is required for Parkin-induced mitochondrial clustering but not mitophagy; VDAC1 is dispensable for both. Autophagy 6, 1090-1106. https://doi.org/10.4161/auto.6.8.13426

49. Narendra, D., Tanaka, A., Suen, D.-F., Youle, R.J., 2008. Parkin is recruited selectively to impaired mitochondria and promotes their autophagy. J Cell Biol 183, 795-803. https://doi.org/10.1083/jcb.200809125

50. Narendra, D.P., Jin, S.M., Tanaka, A., Suen, D.-F., Gautier, C.A., Shen, J., Cookson, M.R., Youle, R.J., 2010. PINK1 Is Selectively Stabilized on Impaired Mitochondria to Activate Parkin. PLOS Biology 8, e1000298. https://doi.org/10.1371/journal.pbio.1000298

51. Nath Neerukonda, S., Mahadev-Bhat, S., Aylward, B., Johnson, C., Charavaryamath, C., Arsenault, R.J., 2018. Kinome analyses of inflammatory responses to swine barn dust extract in human bronchial epithelial and monocyte cell lines. Innate Immun 24, 366-381. https://doi.org/10.1177/1753425918792070 
52. Ney, P.A., 2015. Mitochondrial autophagy: Origins, significance, and role of BNIP3 and NIX. Biochimica et Biophysica Acta (BBA) - Molecular Cell Research, Mitophagy 1853, 2775-2783. https://doi.org/10.1016/j.bbamcr.2015.02.022

53. Nordgren, T.M., Charavaryamath, C., 2018. Agriculture Occupational Exposures and Factors Affecting Health Effects. Curr Allergy Asthma Rep 18, 65. https://doi.org/10.1007/s11882-0180820-8

54. Nugent, S.M.E., Mothersill, C.E., Seymour, C., McClean, B., Lyng, F.M., Murphy, J.E.J., 2007. Increased Mitochondrial Mass in Cells with Functionally Compromised Mitochondria after Exposure to both Direct $\mathrm{Y}$ Radiation and Bystander Factors. rare 168, 134-142. https://doi.org/10.1667/RR0769.1

55. Ott, M., Robertson, J.D., Gogvadze, V., Zhivotovsky, B., Orrenius, S., 2002. Cytochrome c release from mitochondria proceeds by a two-step process. PNAS 99, 1259-1263. https://doi.org/10.1073/pnas.241655498

56. Pagliarini, D.J., Rutter, J., 2013. Hallmarks of a new era in mitochondrial biochemistry. Genes Dev. 27, 2615-2627. https://doi.org/10.1101/gad.229724.113

57. Parisi, M.A., Clayton, D.A., 1991. Similarity of human mitochondrial transcription factor 1 to high mobility group proteins. Science 252, 965-969. https://doi.org/10.1126/science.2035027

58. Pattingre, S., Tassa, A., Qu, X., Garuti, R., Liang, X.H., Mizushima, N., Packer, M., Schneider, M.D., Levine, B., 2005. Bcl-2 Antiapoptotic Proteins Inhibit Beclin 1-Dependent Autophagy. Cell 122, 927-939. https://doi.org/10.1016/j.cell.2005.07.002

59. Picard, M., White, K., Turnbull, D.M., 2013. Mitochondrial morphology, topology, and membrane interactions in skeletal muscle: a quantitative three-dimensional electron microscopy study. J Appl Physiol (1985) 114, 161-171. https://doi.org/10.1152/japplphysiol.01096.2012

60. Pitter, J.G., Maechler, P., Wollheim, C.B., Spät, A., 2002. Mitochondria respond to Ca2+ already in the submicromolar range: correlation with redox state. Cell Calcium 31, 97-104. https://doi.org/10.1054/ceca.2001.0264

61. Qi, L., Sun, X., Li, F.-E., Zhu, B.-S., Braun, F.K., Liu, Z.-Q., Tang, J.-L., Wu, C., Xu, F., Wang, H.-H., Velasquez, L.A., Zhao, K., Lei, F.-R., Zhang, J.-G., Shen, Y.-T., Zou, J.-X., Meng, H.M., An, G.-L., Yang, L., Zhang, X.-D., 2015. HMGB1 Promotes Mitochondrial DysfunctionTriggered Striatal Neurodegeneration via Autophagy and Apoptosis Activation. PLoS One 10. https://doi.org/10.1371/journal.pone.0142901

62. Quinsay, M.N., Lee, Y., Rikka, S., Sayen, M.R., Molkentin, J.D., Gottlieb, R.A., Gustafsson, Å.B., 2010. Bnip3 mediates permeabilization of mitochondria and release of cytochrome c via 
a novel mechanism. Journal of Molecular and Cellular Cardiology 48, 1146-1156. https://doi.org/10.1016/j.yjmcc.2009.12.004

63. Redondo-Horcajo, M., Romero, N., Martínez-Acedo, P., Martínez-Ruiz, A., Quijano, C., Lourenço, C.F., Movilla, N., Enríquez, J.A., Rodríguez-Pascual, F., Rial, E., Radi, R., Vázquez, J., Lamas, S., 2010. Cyclosporine A-induced nitration of tyrosine 34 MnSOD in endothelial cells: role of mitochondrial superoxide. Cardiovasc. Res. 87, 356-365. https://doi.org/10.1093/cvr/cvq028

64. Respiratory Health Hazards in Agriculture, 1998. . Am J Respir Crit Care Med 158, S1-S76. https://doi.org/10.1164/ajrccm.158.supplement_1.rccm1585s1

65. Rikka, S., Quinsay, M.N., Thomas, R.L., Kubli, D.A., Zhang, X., Murphy, A.N., Gustafsson, Å.B., 2011. Bnip3 impairs mitochondrial bioenergetics and stimulates mitochondrial turnover. Cell Death \& Differentiation 18, 721-731. https://doi.org/10.1038/cdd.2010.146

66. Romberger, D.J., Bodlak, V., Von Essen, S.G., Mathisen, T., Wyatt, T.A., 2002. Hog barn dust extract stimulates IL-8 and IL-6 release in human bronchial epithelial cells via PKC activation. Journal of Applied Physiology 93, 289-296. https://doi.org/10.1152/japplphysiol.00815.2001

67. Sahlander, K., Larsson, K., Palmberg, L., 2012. Daily exposure to dust alters innate immunity. PLoS ONE 7, e31646. https://doi.org/10.1371/journal.pone.0031646

68. Senthilselvan, A., Zhang, Y., Dosman, J.A., Barber, E.M., Holfeld, L.E., Kirychuk, S.P., Cormier, Y., Hurst, T.S., Rhodes, C.S., 1997. Positive human health effects of dust suppression with canola oil in swine barns. Am. J. Respir. Crit. Care Med. 156, 410-417. https://doi.org/10.1164/ajrccm.156.2.9612069

69. Sethi, R.S., Schneberger, D., Charavaryamath, C., Singh, B., 2017. Pulmonary innate inflammatory responses to agricultural occupational contaminants. Cell Tissue Res. 367, 627642. https://doi.org/10.1007/s00441-017-2573-4

70. Stefanska, J., Pawliczak, R., 2008. Apocynin: Molecular Aptitudes. Mediators of Inflammation 2008, 1-10. https://doi.org/10.1155/2008/106507

71. Stumbo, A.C., Cortez, E., Rodrigues, C.A., Henriques, M. das G.M.O., Porto, L.C., Barbosa, H.S., Carvalho, L., 2008. Mitochondrial localization of non-histone protein HMGB1 during human endothelial cell-Toxoplasma gondii infection. Cell Biology International 32, 235-238. https://doi.org/10.1016/j.cellbi.2007.08.031

72. Tang, D., Kang, R., Livesey, K.M., Kroemer, G., Billiar, T.R., Van Houten, B., Zeh, H.J., Lotze, M.T., 2011. High-Mobility Group Box 1 Is Essential for Mitochondrial Quality Control. Cell Metabolism 13, 701-711. https://doi.org/10.1016/j.cmet.2011.04.008 
73. Tilokani, L., Nagashima, S., Paupe, V., Prudent, J., 2018. Mitochondrial dynamics: overview of molecular mechanisms. Essays Biochem 62, 341-360. https://doi.org/10.1042/EBC20170104

74. Velde, C.V., Cizeau, J., Dubik, D., Alimonti, J., Brown, T., Israels, S., Hakem, R., Greenberg, A.H., 2000. BNIP3 and Genetic Control of Necrosis-Like Cell Death through the Mitochondrial Permeability Transition Pore. Molecular and Cellular Biology 20, 5454-5468. https://doi.org/10.1128/MCB.20.15.5454-5468.2000

75. Venkataraman, R., Kellum, J.A., Song, M., Fink, M.P., 2002. Resuscitation with Ringer's ethyl pyruvate solution prolongs survival and modulates plasma cytokine and nitrite/nitrate concentrations in a rat model of lipopolysaccharide-induced shock. Shock 18, 507-512. https://doi.org/10.1097/00024382-200212000-00004

76. Vested, A., Basinas, I., Burdorf, A., Elholm, G., Heederik, D.J.J., Jacobsen, G.H., Kolstad, H.A., Kromhout, H., Omland, Ø., Sigsgaard, T., Thulstrup, A.M., Toft, G., Vestergaard, J.M., Wouters, I.M., Schlünssen, V., 2019. A nationwide follow-up study of occupational organic dust exposure and risk of chronic obstructive pulmonary disease (COPD). Occup Environ Med 76, 105-113. https://doi.org/10.1136/oemed-2018-105323

77. Wai, T., Langer, T., 2016. Mitochondrial Dynamics and Metabolic Regulation. Trends in Endocrinology \& Metabolism 27, 105-117. https://doi.org/10.1016/j.tem.2015.12.001

78. West, A.P., Khoury-Hanold, W., Staron, M., Tal, M.C., Pineda, C.M., Lang, S.M., Bestwick, M., Duguay, B.A., Raimundo, N., MacDuff, D.A., Kaech, S.M., Smiley, J.R., Means, R.E., Iwasaki, A., Shadel, G.S., 2015. Mitochondrial DNA stress primes the antiviral innate immune response. Nature 520, 553-557. https://doi.org/10.1038/nature14156

79. Wunschel, J., Poole, J.A., 2016. Occupational agriculture organic dust exposure and its relationship to asthma and airway inflammation in adults. J Asthma 53, 471-477. https://doi.org/10.3109/02770903.2015.1116089

80. Yu, J., Nagasu, H., Murakami, T., Hoang, H., Broderick, L., Hoffman, H.M., Horng, T., 2014. Inflammasome activation leads to Caspase-1-dependent mitochondrial damage and block of mitophagy. Proc Natl Acad Sci $U$ S A 111, 15514-15519. https://doi.org/10.1073/pnas.1414859111

81. Yu, Y.-M., Kim, J.-B., Lee, K.-W., Kim, S.Y., Han, P.-L., Lee, J.-K., 2005. Inhibition of the cerebral ischemic injury by ethyl pyruvate with a wide therapeutic window. Stroke 36, 22382243. https://doi.org/10.1161/01.STR.0000181779.83472.35 
960

961

962

963

964

965

966

967

968

969

970

971

972

973

974

975

976

977

978

979

980

981

983

984

985

83

982

82. Zhang, Q., Raoof, M., Chen, Y., Sumi, Y., Sursal, T., Junger, W., Brohi, K., Itagaki, K., Hauser, C.J., 2010. Circulating mitochondrial DAMPs cause inflammatory responses to injury. Nature 464, 104-107. https://doi.org/10.1038/nature08780

83. Zorov, D.B., Juhaszova, M., Sollott, S.J., 2014. Mitochondrial Reactive Oxygen Species (ROS) and ROS-Induced ROS Release. Physiol Rev 94, 909-950. https://doi.org/10.1152/physrev.00026.2013

6

67

8

9

90

71

2

73

74

75

76

79

81




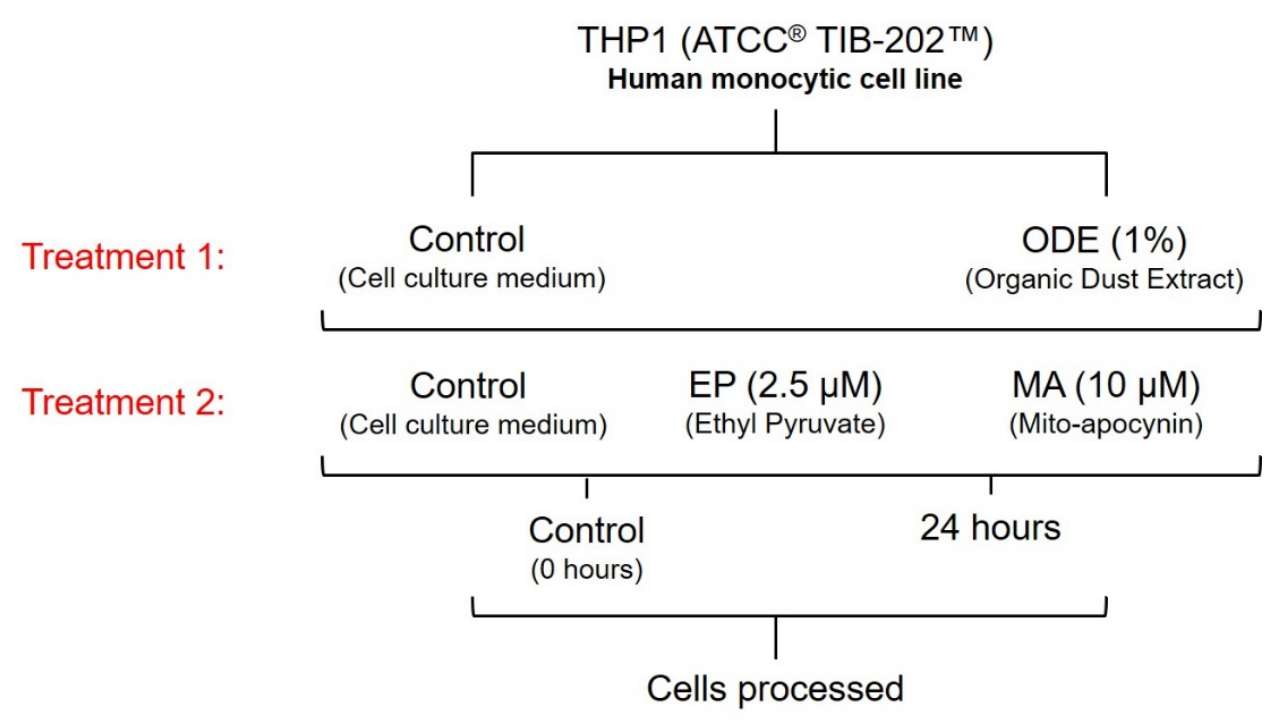

Figure 1. ODE exposure of THP1 cells and antioxidant treatment. THP1 cells were treated 989 with either media (control) or ODE (treatment 1) followed by either media, EP or MA (treatment 990 2). Cells were processed for various assays at 0 (control), and 24 hours.

991

992

993

994

995

996

997 
(a)

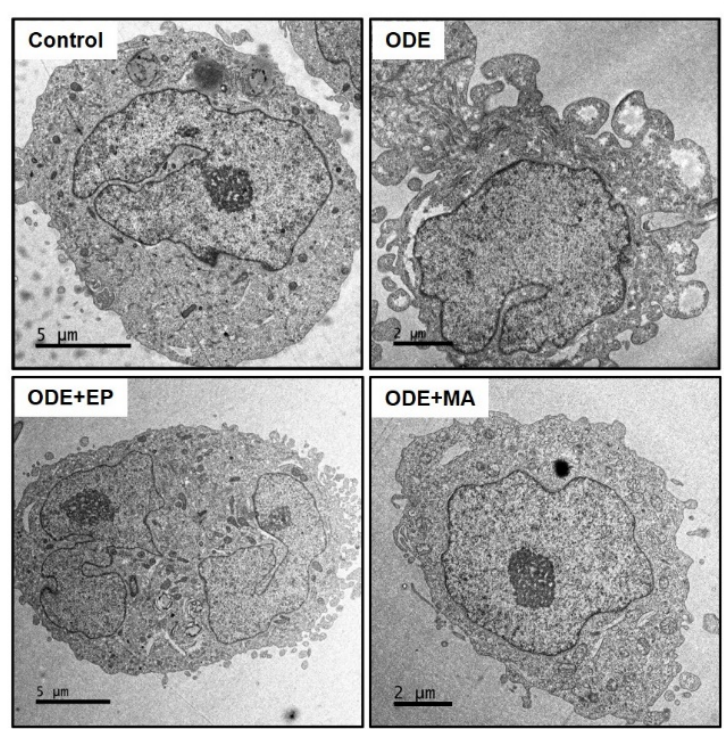

(c)

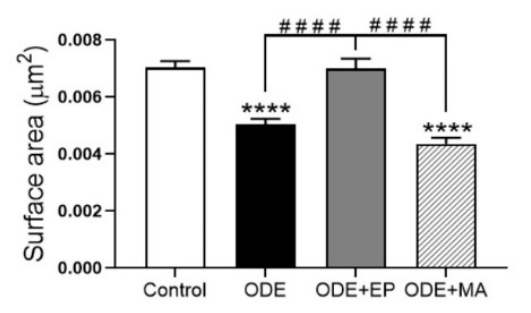

(e)

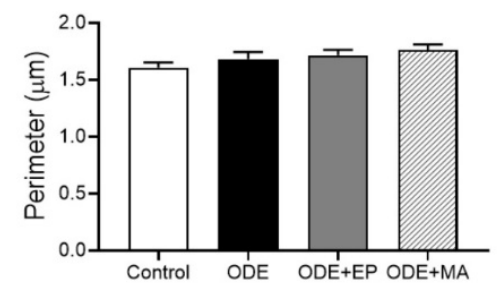

(b)

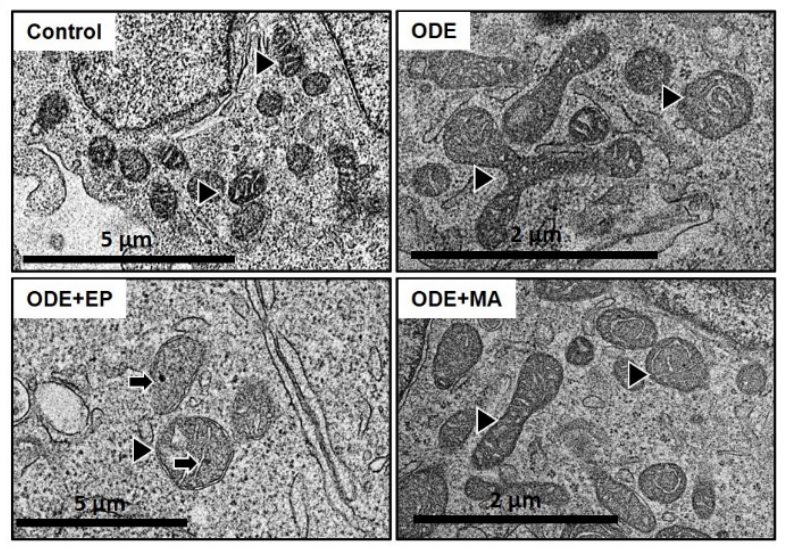

(d)

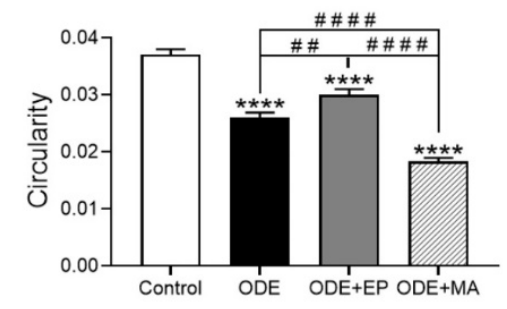

(f)

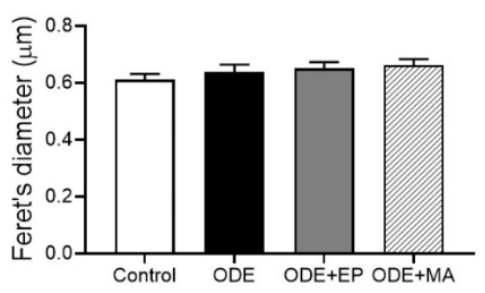

Figure 2. ODE exposure induces differentiation of THP1 cells and changes in mitochondrial

1000

1001

1002

1003

1004

1005

1006

1007

1008

1009 morphology. Transmission electron microscopy (TEM) of THP1 cells treated with ODE and antioxidant therapy for 24 hours shows changes in cellular and mitochondrial morphology at the ultrastructural level. Compared to controls, cells undergo differentiation into activated macrophages, with increased vacuolation and pseudopod formation on treatment with ODE (1\%; a). Scale bar, 2-5 $\mu \mathrm{m}$. A number of mitochondria show changes in morphology (fission/fusion) and swelling (b), along with presence of calcium sequestration bodies within the mitochondrial matrix in cells co-treated with $2.5 \mu \mathrm{M}$ of EP, and noticeably healthier mitochondria with some morphological changes (fission/fusion) in cells co-treated with $10 \mu \mathrm{M}$ of MA. Morphological parameters of mitochondria on treatment was analyzed by ImageJ (c-f). Significant change in the 
surface area (c) and circularity (d) indicative of mitochondrial fragmentation, and no change in perimeter (e) and the feret's diameter (f). Data analyzed via one-way ANOVA with Tukey's

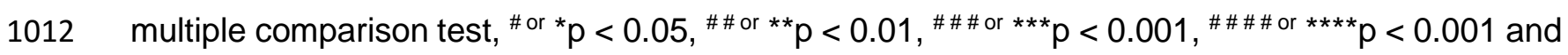
1013 are represented as Mean \pm SEM with $n=126$ mitochondria/treatment.

(a)

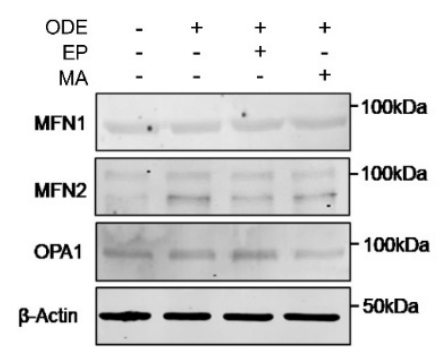

(c)

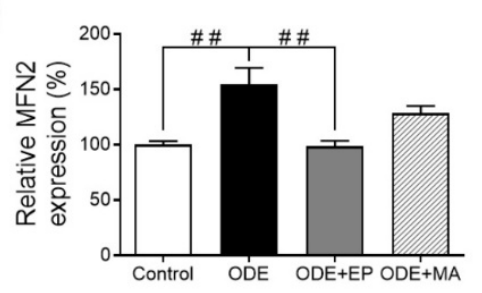

(e)
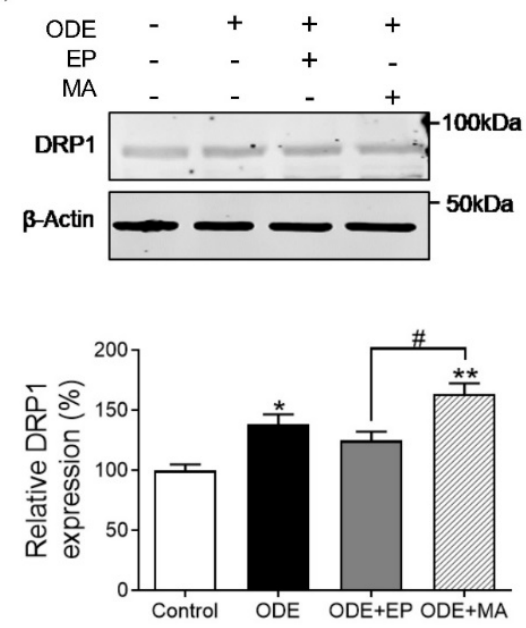

(b)

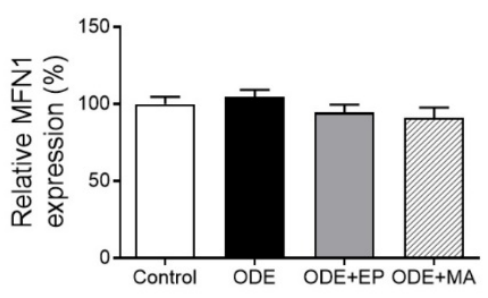

(d)

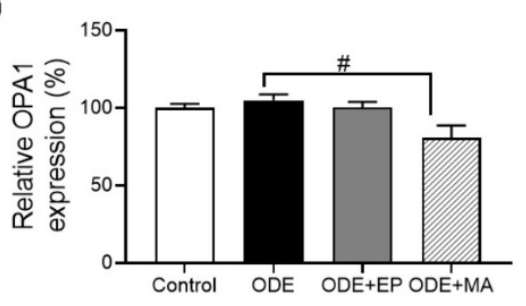

(f)

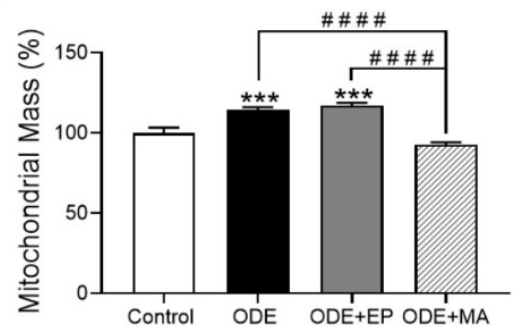

1015

Figure 3. ODE exposure induces fusion of mitochondria in response to stress. Immunoblotting of whole cell lysates of THP1 cells, treated with ODE and antioxidant therapy for

1018 24 hours, was performed to detect mitochondrial fusion and fission proteins. Compared to controls, ODE (1\%) treated cells showed minimal changes in the expression of MFN1/2 and OPA1 (a-d), while OPA1 expressions is significantly decreased on exposure to $10 \mu \mathrm{M}$ of MA. ODE

1021 treated cells showed an increase in DRP1 expression, whereas co-treatment with $10 \mu \mathrm{M}$ of MA, 
significantly upregulates DRP 1 expression and co-treatment with $2.5 \mu \mathrm{M}$ of EP downregulates DRP1 comparable to control (e). Mitochondrial mass was measured by Mito-Tracker staining and data showed a significant increase in the mass with ODE (1\%) treatment, while $10 \mu \mathrm{M}$ of MA significantly reduced the mitochondrial mass to the baseline (control) levels (f). For all western blots, samples were derived from the same experiment and were processed in parallel. All protein bands were normalized over $\beta$-actin (37 kD) and percentage intensity relative to control analyzed. All data analyzed via one-way ANOVA with Tukey's multiple comparison test, \# or *p $<0.05$, \#\# or

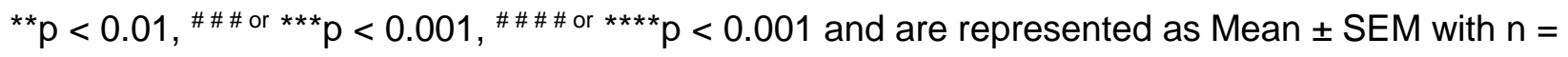
3-6/treatment.

(a)

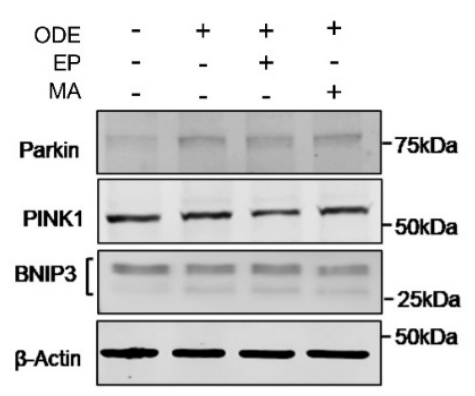

(c)

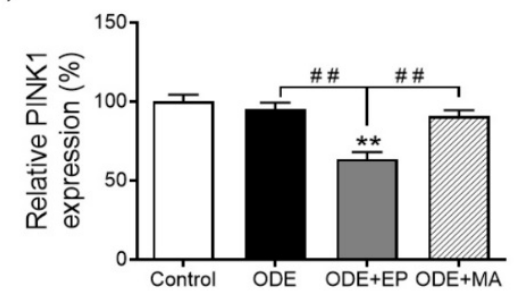

(b)

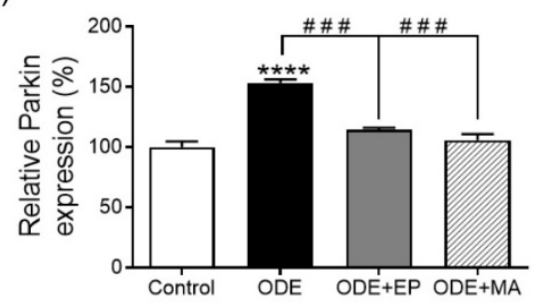

(d)

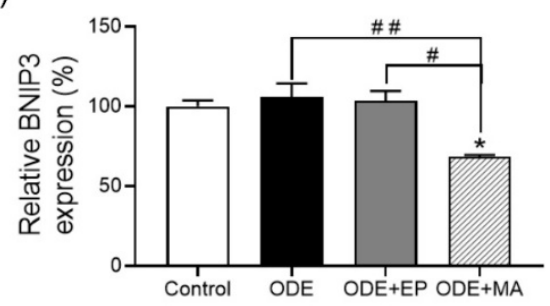

1031

1032

1033

1034

1035

1036

1037

1038

1039

1040

1041

Figure 4. ODE exposure induces selective targeting of mitochondria for autophagy (mitophagy). Immunoblotting of whole cell lysates of THP1 cells, treated with ODE and antioxidant therapy for 24 hours, was performed to detect expression of mitophagy markers. Compared to controls, cells treated with ODE (1\%) showed increase in the expression of Parkin (a-b), while expression of PINK1 remains relatively constant (c). Co-treatment with $10 \mu \mathrm{M}$ of MA significantly decreased Parkin and BNIP3 expressions (b \& d). Co-treatment with $2.5 \mu \mathrm{M}$ of EP significantly decreased PINK1 and Parkin expressions, while having no impact on BNIP3 (a-d). For all western blots, samples were derived from the same experiment and were processed in parallel. All protein bands were normalized over $\beta$-actin (37 kD) and percentage intensity relative to control analyzed. All data analyzed via one-way ANOVA with Tukey's multiple comparison test, 
$1043 \pm$ SEM with $n=3 /$ treatment.

(a)

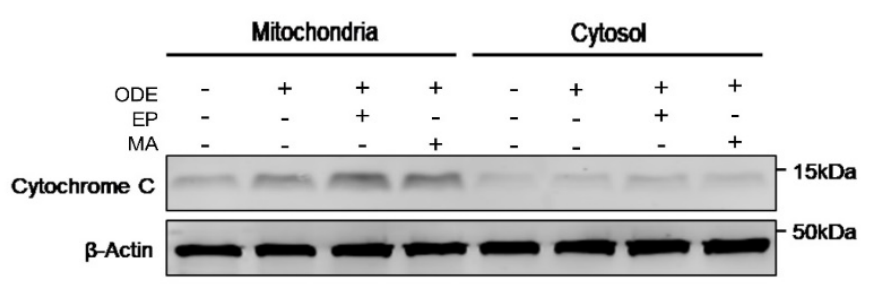

(b)

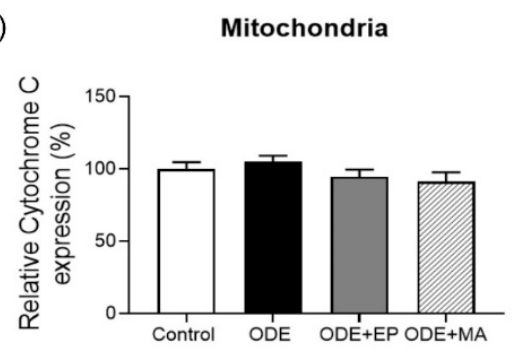

(d)
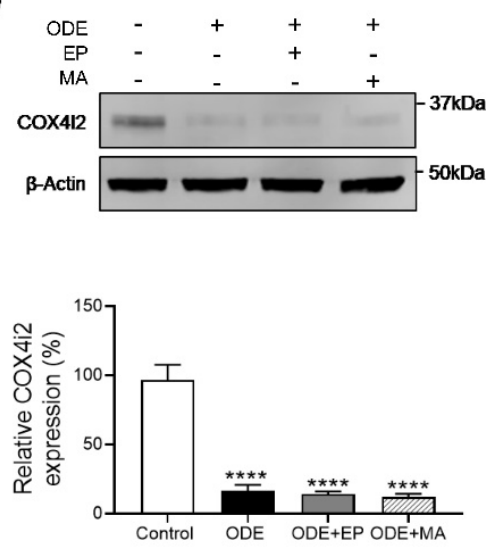

(f)

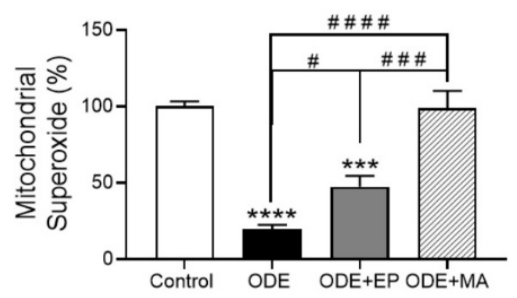

(c)

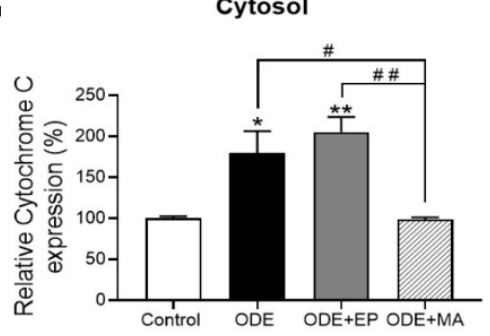

(e)
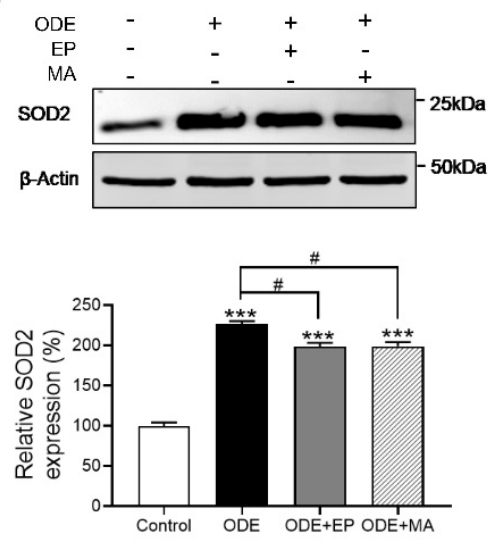

(g)

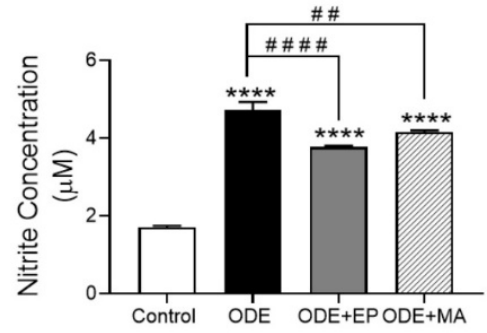

1047 Figure 5. Mitoapocyanin treatment decreases ODE-induced Cytochrome C release and 1048 markedly increases SOD2 expression in the cytosol. Immunoblotting of mitochondrial and 1049 mitochondria-free cytosolic fractions of THP1 cells, treated with ODE and antioxidant therapy for 
105024 hours, was performed to detect the presence of Cytochrome $C$ and expression of lung-specific 1051 isoform of COX, COX4i2, on ODE exposure. ODE (1\%) and $2.5 \mu \mathrm{M}$ of EP co-treated cells showed 1052 a significant increase in Cytochrome $C$ in the cytosol ( $a \& c)$, while treatment with $10 \mu \mathrm{M}$ of MA 1053 downregulated Cytochrome C comparable to control (c). No change in levels of mitochondrial 1054 Cytochrome $\mathrm{C}$ was observed for all treatments (b). ODE (1\%) treated cells showed a significant 1055 decrease in COX4i2 expression (d), while superoxide dismutase 2 (SOD2) increased compared 1056 to controls and abrogated when treated with $10 \mu \mathrm{M}$ of MA and $2.5 \mu \mathrm{M}$ of EP (e). MitoSOX assay 1057 performed showed a decrease in superoxide anions (SOX) on ODE (1\%) exposure, while levels 1058 on treatment with treated with $10 \mu \mathrm{M}$ of MA was comparable to control (f). Griess assay was 1059 performed to measure the amount of nitrite levels secreted. ODE (1\%) treated cells were 1060 observed to secrete elevated levels of nitrite (g). Co-treatment with $2.5 \mu \mathrm{M}$ of EP or $10 \mu \mathrm{M}$ of MA 1061 decreased nitrite levels. For all western blots, samples were derived from the same experiment 1062 and were processed in parallel. All protein bands were normalized over $\beta$-actin (37 kD) and 1063 percentage intensity relative to control analyzed. All data analyzed via one-way ANOVA with

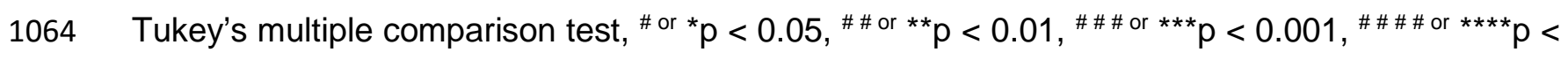
10650.001 and are represented as Mean \pm SEM with $n=3-6 /$ treatment. 
(a)

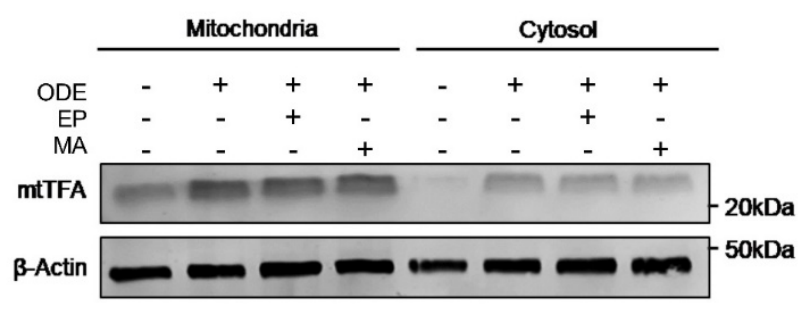

(b)

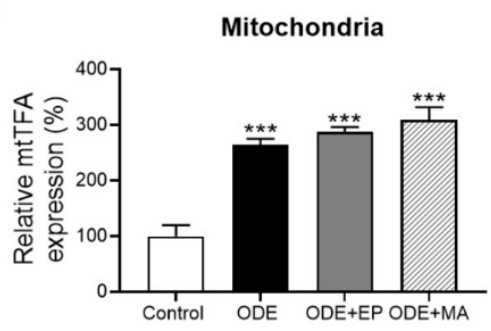

(d)

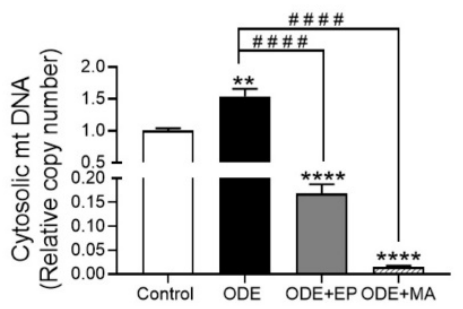

(e)

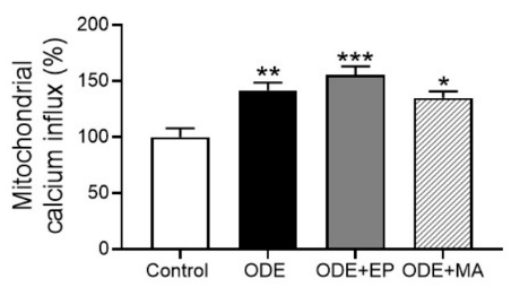

(c)

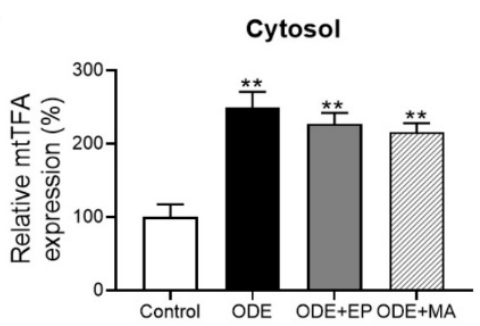

(f)
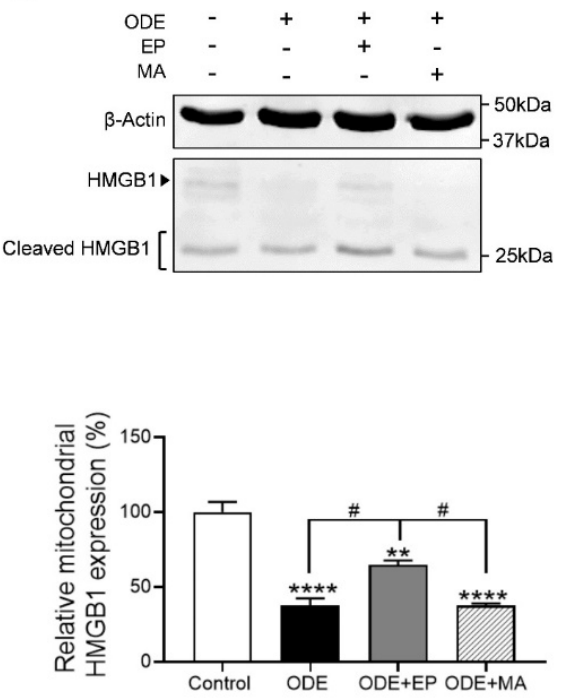

Figure 6. ODE exposure markedly increases secretion of mitochondrial DAMPs into the cytosol. Immunoblotting of mitochondrial and mitochondria-free cytosolic fractions of THP1 cells, treated with ODE and antioxidant therapy for 24 hours, was performed to detect expression of mitochondrial transcription factor A (mtTFA). ODE (1\%) treated cells increase mtTFA expression

1072 in the cytosol, while treatment with $10 \mu \mathrm{M}$ of MA increased mtTFA in the mitochondrial matrix (a). 1073 Mitochondrial DNA leakage into the cytosol analyzed via qPCR was higher in the cytosol on ODE 1074 (1\%) exposure which was decreased by $10 \mu \mathrm{M}$ of MA (d). Intra-mitochondrial calcium levels 1075 measured by Rhod 2AM staining, showed increased calcium levels on ODE (1\%) exposure, which 1076 remained unaffected by $10 \mu \mathrm{M}$ of MA (e). Immunoblotting of mitochondrial fraction of THP1 cells 
was performed to measure mitochondrial HMGB1. ODE (1\%) treatment showed a decrease in HMGB1, while EP treatment increased the HMGB1 compared to either ODE or MA (f). For all western blots, samples were derived from the same experiment and were processed in parallel. All protein bands were normalized over $\beta$-actin $(37 \mathrm{kD})$ and percentage intensity relative to control analyzed. All data analyzed via one-way ANOVA with Tukey's multiple comparison test, \# or *p <

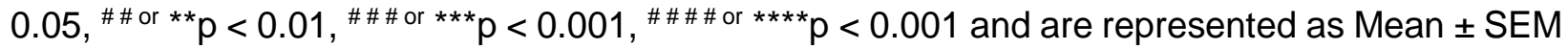
with $n=3-6 /$ treatment.

(a)

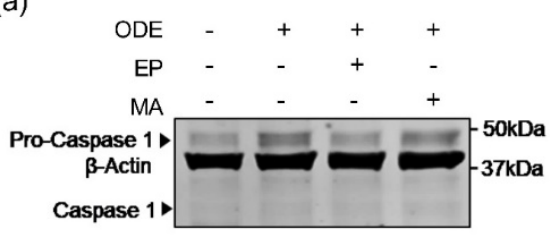

(b)

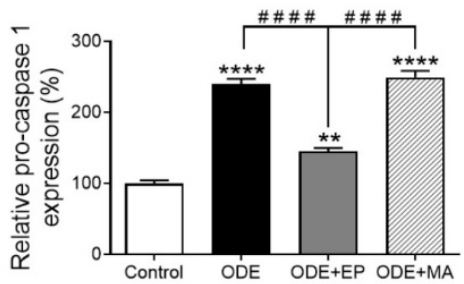

(c)

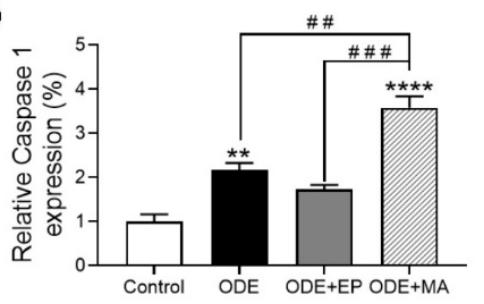

(d)

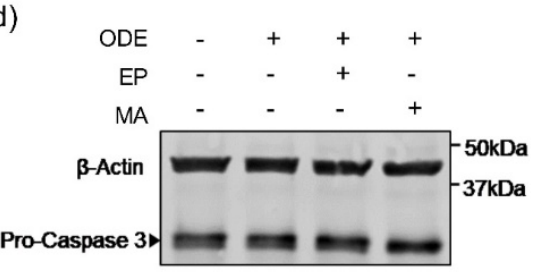

(e)

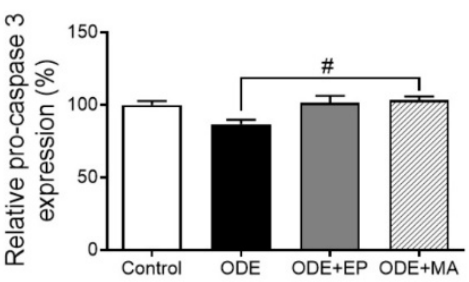

1084

1085

1086

1087

1088

1089

1090

1091

1092

1093

1094

1095
Figure 7. ODE exposure increases expression of Caspase 1 consistent in inflammatory conditions. Immunoblotting of whole cell lysates of THP1 cells, treated with ODE and antioxidant therapy for 24 hours, was performed to detect the expression of caspase 1 and 3. Cells treated with ODE (1\%) and $10 \mu \mathrm{M}$ of MA increase in pro-caspase 1, along with the cleaved caspase 1 p10, while co-treatment with $2.5 \mu \mathrm{M}$ of EP decreased expression comparable to control (a-c). No significant difference observed with pro-caspase 3 and absence of cleaved caspase 3 (d \& e). For all western blots, samples were derived from the same experiment and were processed in parallel. All protein bands were normalized over $\beta$-actin (37 kD) and percentage intensity relative to control analyzed. All data analyzed via one-way ANOVA with Tukey's multiple comparison test,

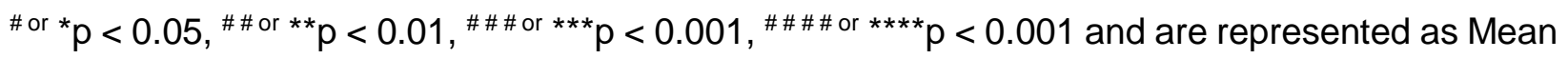
\pm SEM with $n=3 /$ treatment. 
(a)
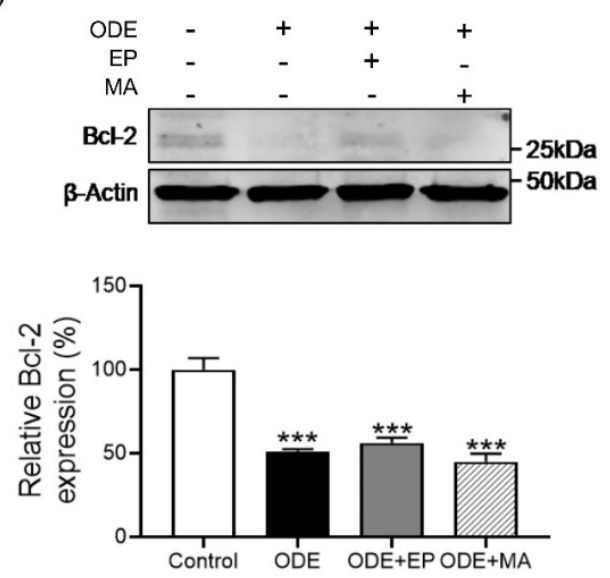

(c)

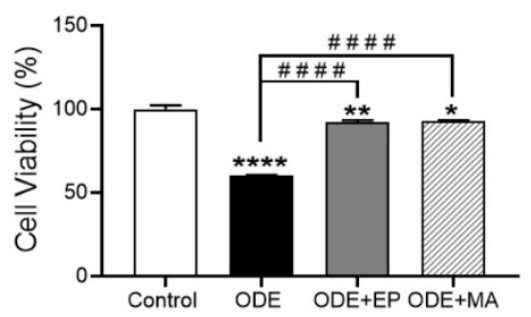

(b)
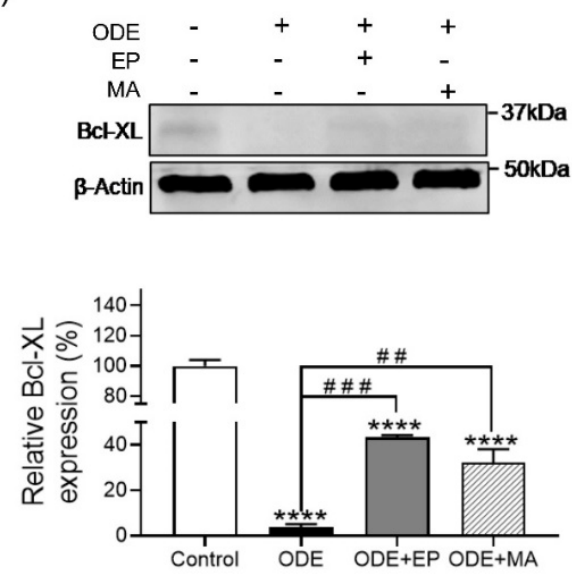

1097 Figure 8. Mitochondrial targeted antioxidant treatment has no effect $\mathrm{Bcl}-2$ and $\mathrm{Bcl}-\mathrm{XL}$

1098 expression. Immunoblotting of whole cell lysates of THP1 cells, treated with ODE and antioxidant 1099 therapy for 24 hours, was performed to observe expression of $\mathrm{Bcl}-2$ and $\mathrm{Bcl}-\mathrm{XL}$. ODE (1\%) 1100 treatment decreased $\mathrm{Bcl}-2$ and $\mathrm{Bcl}-\mathrm{XL}(\mathrm{a}-\mathrm{c})$, while co-treatment with $10 \mu \mathrm{M}$ of MA increased Bcl1101 XL comparably higher than ODE (1\%; b). MTT assay to measure cell viability showed increased 1102 viability on co-treatment with $2.5 \mu \mathrm{M}$ of EP or $10 \mu \mathrm{M}$ of MA compared to ODE (c). For all western 1103 blots, samples were derived from the same experiment and were processed in parallel. All protein 1104 bands were normalized over $\beta$-actin (37 kD) and percentage intensity relative to control analyzed. 1105 All data analyzed via one-way ANOVA with Tukey's multiple comparison test, \# or *p < 0.05, \#\# or

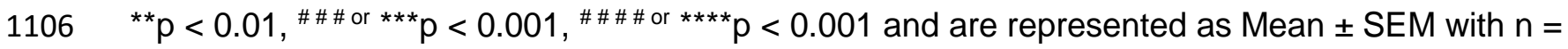
1107 3-6/treatment. 